
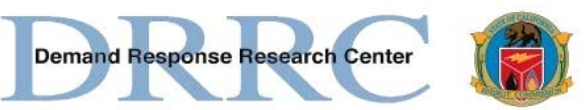

LBNL-62226

\title{
Demand Responsive Lighting: A Scoping Study
}

\author{
Francis Rubinstein \\ Lawrence Berkeley National Laboratory \\ Sila Kiliccote \\ Lawrence Berkeley National Laboratory
}

1 Cyclotron Rd.

Building 90R3111

Berkeley CA 94720

January 3, 2007

This work described in this report was coordinated by the Demand Response Research Center and funded by the California Energy Commission, Public Interest Energy Research Program, under Work for Others Contract No. 500-03-026 and by the U.S. Department of Energy under Contract No. DE-AC02$05 \mathrm{CH} 11231$. 


\section{Legal Notice}

This report was prepared as a result of work sponsored by the California Energy Commission (Energy Commission). It does not necessarily represent the views of the Energy Commission, its employees, or the State of California. The Energy Commission, the State of California, its employees, contractors, and subcontractors make no warrant, express or implied, and assume no legal liability for the information in this report; nor does any party represent that the use of this information will not infringe upon privately owned rights. This report has not been approved or disapproved by the Energy Commission, nor has the Energy Commission passed upon the accuracy or adequacy of this information in this report. 


\section{Acknowledgment and Citation Page}

This work described in this report was coordinated by the Demand Response Research Center and funded by the California Energy Commission (CEC), Public Interest Energy Research (PIER) Program, under Work for Others Contract No. 500-03-026 and by the U.S. Department of Energy under Contract No. DE-AC02-05CH11231. 


\section{Preface}

The Public Interest Energy Research (PIER) Program supports public interest energy research and development that will help improve the quality of life in California by bringing environmentally safe, affordable, and reliable energy services and products to the marketplace.

The PIER Program, managed by the California Energy Commission (Commission), annually awards up to $\$ 62$ million to conduct the most promising public interest energy research by partnering with Research, Development, and Demonstration (RD\&D) organizations, including individuals, businesses, utilities, and public or private research institutions.

PIER funding efforts are focused on the following six RD\&D program areas:

- Buildings End-Use Energy Efficiency

- Industrial/Agricultural/Water End-Use Energy Efficiency

- Renewable Energy

- Environmentally-Preferred Advanced Generation

- Energy-Related Environmental Research

- Energy Systems Integration

What follows is the final report for the Dimmable Ballasts Project, 500-03-026 Task 4-4, conducted by Lawrence Berkeley National Laboratory. The report is entitled "Demand Response Lighting: A Scoping Study". This project contributes to the Energy Systems Integration Program.

For more information on the PIER Program, please visit the Commission's Web site at:

http://www.energy.ca.gov/research/index.html or contact the Commission's Publications Unit at 916-654-5200. 


\section{Table of Contents}

Abstract

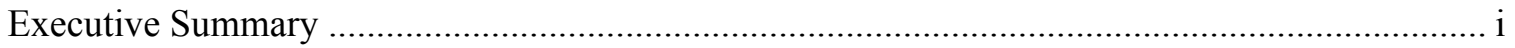

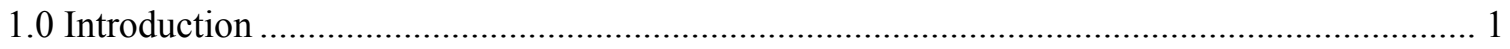

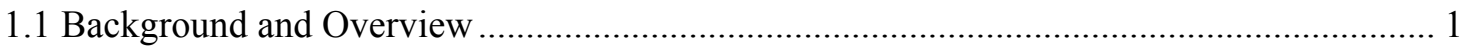

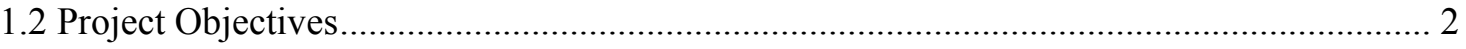

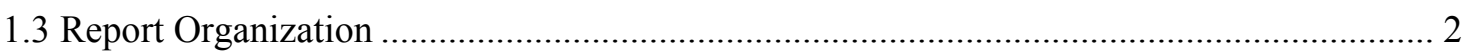

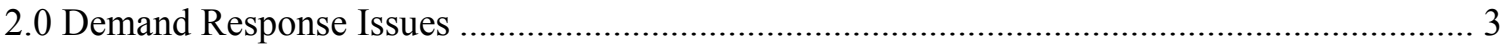

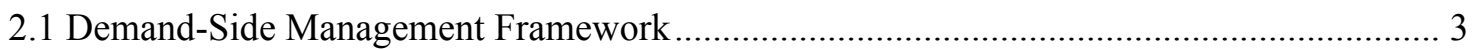

3.0 Previous Experience with Demand Responsive Lighting Systems ..................................... 6

3.1. Load Shedding at the Watergate Office Building, Emeryville, CA ................................. 6

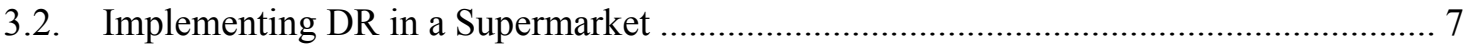

4.0 Demand and Energy Consumption Patterns of California Commercial Buildings.............. 10

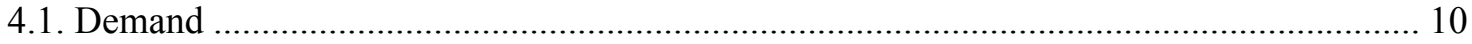

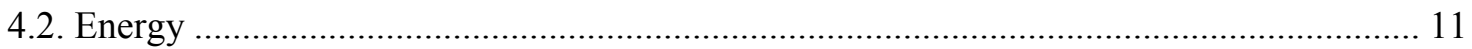

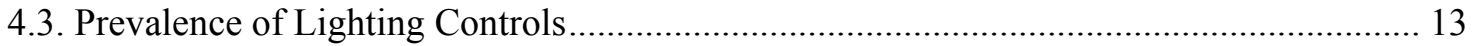

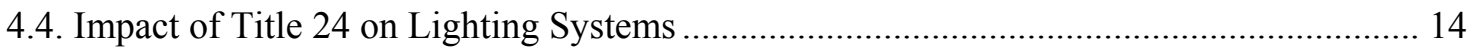

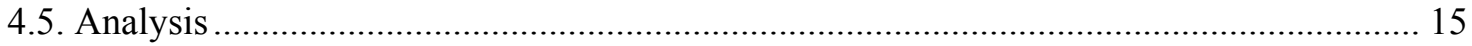

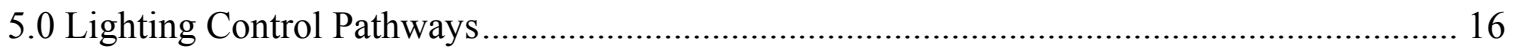

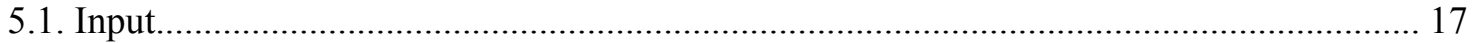

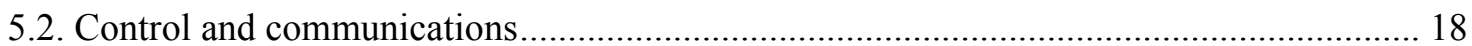

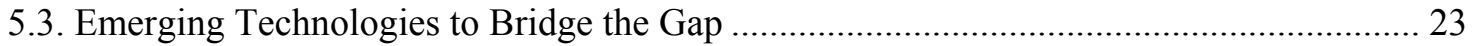

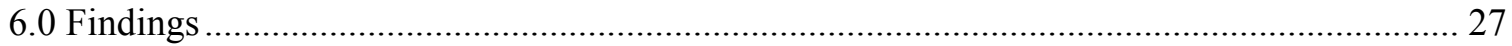

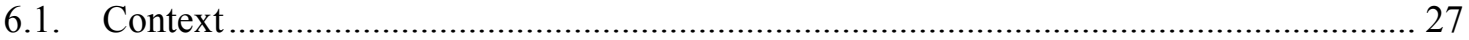

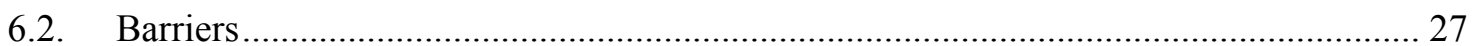

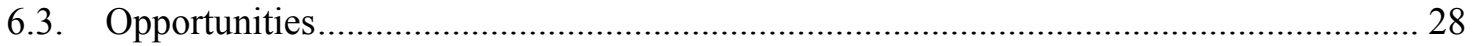

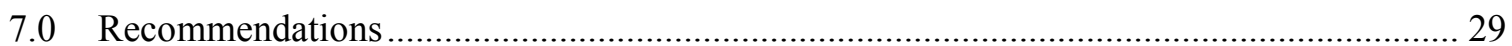

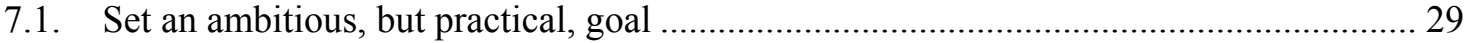

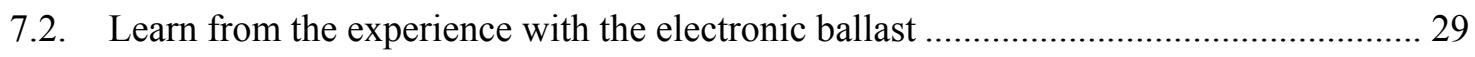

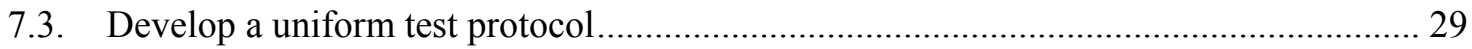

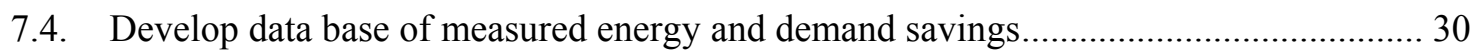

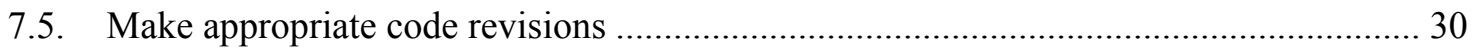

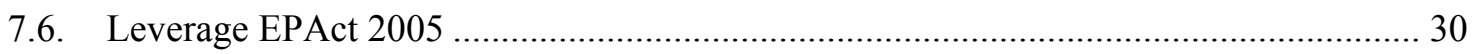

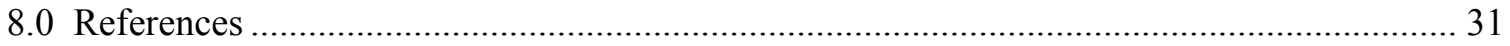

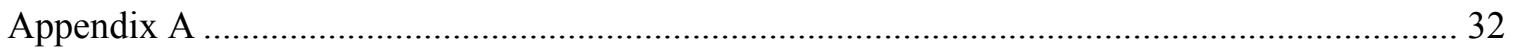

State-of-the-Art Review of the Performance Characteristics of Dimming Ballasts ...................... 32

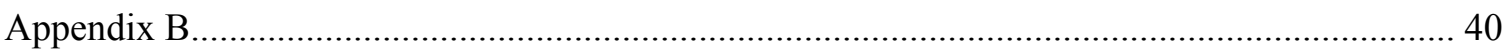

Projected Energy Savings and Market Potential for Wireless Lighting Controls in the State of

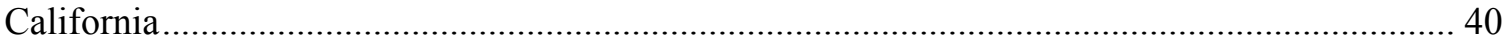

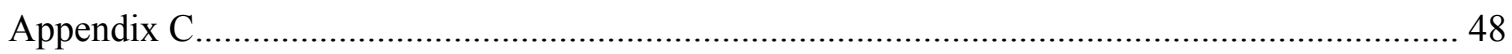




\section{List of Figures}

Figure 1: California commercial sector electrical demand (megawatts) for 1999 Summer Peak broken down by major end-use (left). Dimming all commercial lighting to 25\% (right) could theoretically reduce total commercial peak by $7 \mathrm{GW}$.

Figure 2: Demand profile of various demand response methods 1

Figure 3: Flow diagram for DR Decisions for Lighting Systems

Figure 4. PGE-funded lighting controls research in 1991 was the first to examine the effectiveness of using dimmable lighting to achieve significant demand reduction (aka demand response).

Figure 5: Total electric power in supermarket during two step load shed experiment.

Figure 6: Overhead lighting at normal (left) and in load shed mode (right). In load shed mode, about 50\%

of the rows of lamps are switched off.

Figure 7: Maximum Demands Savings Intensity due to Lighting System Response

Figure 8: End Use Loads for California Commercial Sector on 2003 Peak Day [Source: CEC 2003 Data]

Figure 9: Lighting Energy Usage for Commercial Building Lighting in California $2000 \_12$

Figure 10: Commercial Indoor Lighting Energy Use ___ 13

Figure 11: Lighting Controls Penetration Compared with HVAC Controls in Commercial Buildings _ 14

Figure 12: A Framework for Lighting Control

Figure 14: Wiring diagram showing how 0-10 VDC analog ballasts are wired to a low voltage control loop formed by the violet and grey wires. 21

Figure 15: Wiring diagram showing how powerline controlled ballasts are wired to a wall dimmer.__ 22

Figure 16: Wiring diagram for a typical DALI ballast. Multiple DALI ballasts are wired in parallel to the DALI control leads. Unlike 0-10 VDC analog dimming ballasts, DALI ballasts are individually addressable.

Figure 17: Description of the GE Wireless Energy Management System

Figure 18: Pathways concept as applied to Ganahl Lumber Company tests 25

Figure 19: Pathways concept as applied to Target Company Automated Critical Peak Pricing Tests _ 26 


\section{List of Tables}

Table 1: Energy efficiency, daily load management and DR 3

Table 2. Different configurations for applying controllers to existing buildings 20 


\begin{abstract}
The objective of this scoping study is: 1) to identify current market drivers and technology trends that can improve the demand responsiveness of commercial building lighting systems and 2) to quantify the energy, demand and environmental benefits of implementing lighting demand response and energy-saving controls strategies Statewide. Lighting systems in California commercial buildings consume $30 \mathrm{GWh}$. Lighting systems in commercial buildings often waste energy and unnecessarily stress the electrical grid because lighting controls, especially dimming, are not widely used. But dimmable lighting equipment, especially the dimming ballast, costs more than non-dimming lighting and is expensive to retrofit into existing buildings because of the cost of adding control wiring. Advances in lighting industry capabilities coupled with the pervasiveness of the Internet and wireless technologies have led to new opportunities to realize significant energy saving and reliable demand reduction using intelligent lighting controls. Manufacturers are starting to produce electronic equipment -- lighting-application specific controllers (LAS controllers) -- that are wirelessly accessible and can control dimmable or multilevel lighting systems obeying different industry-accepted protocols. Some companies make controllers that are inexpensive to install in existing buildings and allow the power consumed by bi-level lighting circuits to be selectively reduced during demand response curtailments. By intelligently limiting the demand from bi-level lighting in California commercial buildings, the utilities would now have an enormous 1 GW demand shed capability at hand. By adding occupancy and light sensors to the remotely controllable lighting circuits, automatic controls could harvest an additional $1 \mathrm{BkWh} / \mathrm{yr}$ savings above and beyond the savings that have already been achieved. The lighting industry's adoption of DALI as the principal wired digital control protocol for dimming ballasts and increased awareness of the need to standardize on emerging wireless technologies are evidence of this transformation. In addition to increased standardization of digital control protocols controller capabilities, the lighting industry has improved the performance of dimming lighting systems over the last two years. The system efficacy of today's current dimming ballasts is approaching that of non-dimming program start ballasts.

The study finds that the benefits of applying digital controls technologies to California's unique commercial buildings market are enormous. If California were to embark on an concerted 20 year program to improve the demand responsiveness and energy efficiency of commercial building lighting systems, the State could avoid adding generation capacity, improve the elasticity of the grid, save Californians billion of dollars in avoided energy charges and significantly reduce greenhouse gas emissions.
\end{abstract}




\section{Executive Summary}

\section{Objective}

The objective of this scoping study is: 1) to identify current market drivers and technology trends that can improve the demand responsiveness of commercial building lighting systems and 2) to quantify the energy, demand and environmental benefits of implementing lighting demand response and energy-saving controls strategies Statewide.

\section{Lighting Energy Consumption}

Lighting systems in California commercial buildings consume 30 GWh. Large offices, retail, hospital, miscellaneous and food stores make up nearly $60 \%$ of the total 6 billion square feet of commercial floorspace and use about $75 \%$ of the total lighting energy. Commercial lighting demand is largely coincident with total Statewide peak demand and on peak days is responsible for $30 \%$ of the total demand during the 2:00 PM- 5:00 PM summer peak (compared to $32 \%$ for air conditioning). Lighting, therefore, has a major impact on electricity demand in commercial buildings.

\section{Dimmable Lighting Underutilized}

Lighting systems in commercial buildings often waste energy and unnecessarily stress the electrical grid because lighting controls, especially dimming, are not widely used. By drawing less power from the grid when electricity costs are highest, dimming ballasts are an enabling technology that allows building lighting loads to become more elastic. But dimmable lighting equipment, especially the dimming ballast, costs more than non-dimming lighting and is expensive to retrofit into existing buildings because of the cost of adding control wiring.

Opportunities for New Technologies

Recent renewed concern for electricity disruptions and power outages has stimulated the industry to re-examine and re-design their dimming product lines to implement demand response and energy efficiency measures. Advances in lighting industry capabilities coupled with the pervasiveness of the Internet and wireless technologies have led to new opportunities to realize significant energy saving and reliable demand reduction using intelligent lighting controls. Manufacturers are starting to produce electronic equipment -- lighting-application specific controllers (LAS controllers) -- that are wirelessly accessible and can control dimmable or multilevel lighting systems obeying different industry-accepted protocols. LAS controllers are particularly well-suited to retrofitting where it is not cost-effective to add wiring to communicate with downstream lights.

The study finds that controllers are becoming available that can operate dimming ballasts and multi-level lighting using 1) low-voltage digital, 2) powerline, 3) powerline-carrier or 4) pure wireless communication protocols. System integrators will soon be able to specify entire solution pathways that couple an appropriate LAS controller with a downstream lighting system and appropriate communications protocol. A major purpose of the study is to detail the major pathways that system integrators can take to cost-effectively implement demand response and energy efficiency control strategies in commercial lighting systems, both for existing buildings and new construction.

\section{Bi-Level Lighting Saves Energy}

Because of Title 24's influence, lighting systems in California have some unique characteristics, especially in how they are electrically wired for bi-level lighting. There are major opportunities to tap the huge reservoir of potential energy and demand savings inherent in the State's wiring infrastructure.

One of the favorable long-term consequences of California's Title 24 Building Standard is that a significant proportion of standing commercial building stock enjoys bi-level switching. Bi-level 
switching is defined as a manual control that provides two levels of lighting power in a space (not including off). Recent studies found a $15 \%$ average energy savings from occupants using bi-level switching in offices, retail stores and schools. Thus California has already been saving \$100-150 million/yr because of bi-level switching. Some companies make controllers that are inexpensive to install in existing buildings and allow the power consumed by bi-level lighting circuits to be selectively reduced during demand response curtailments. By intelligently limiting the demand from bi-level lighting in California commercial buildings, the utilities would now have an enormous $1 \mathrm{GW}$ demand shed capability at hand. By adding occupancy and light sensors to the remotely controllable lighting circuits, automatic controls could harvest an additional $1 \mathrm{BkWh} / \mathrm{yr}$ savings above and beyond the savings that have already been achieved.

Progress in Standardization for Digital Lighting Controls

One of the technology trends identified in the study is the increased digitization of the lighting controls industry. The lighting industry's adoption of DALI as the principal wired digital control protocol for dimming ballasts and increased awareness of the need to standardize on emerging wireless technologies are evidence of this transformation.

Improvements in Dimming Ballast Performance

In addition to increased standardization of digital control protocols controller capabilities, the lighting industry has improved the performance of dimming lighting systems over the last two years. The system efficacy of today's current dimming ballasts is approaching that of nondimming program start ballasts.

\section{Significant Energy and Environmental Benefits}

The study finds that the benefits of applying digital controls technologies to California's unique commercial buildings market are enormous. If California were to embark on an concerted 20 year program to improve the demand responsiveness and energy efficiency of commercial building lighting systems, the State could avoid adding generation capacity, improve the elasticity of the grid, save Californians billion of dollars in avoided energy charges and significantly reduce greenhouse gas emissions. 


\section{Projected I mpact of Wireless Lighting Controls on CA Commercial Lighting Energy Consumption}

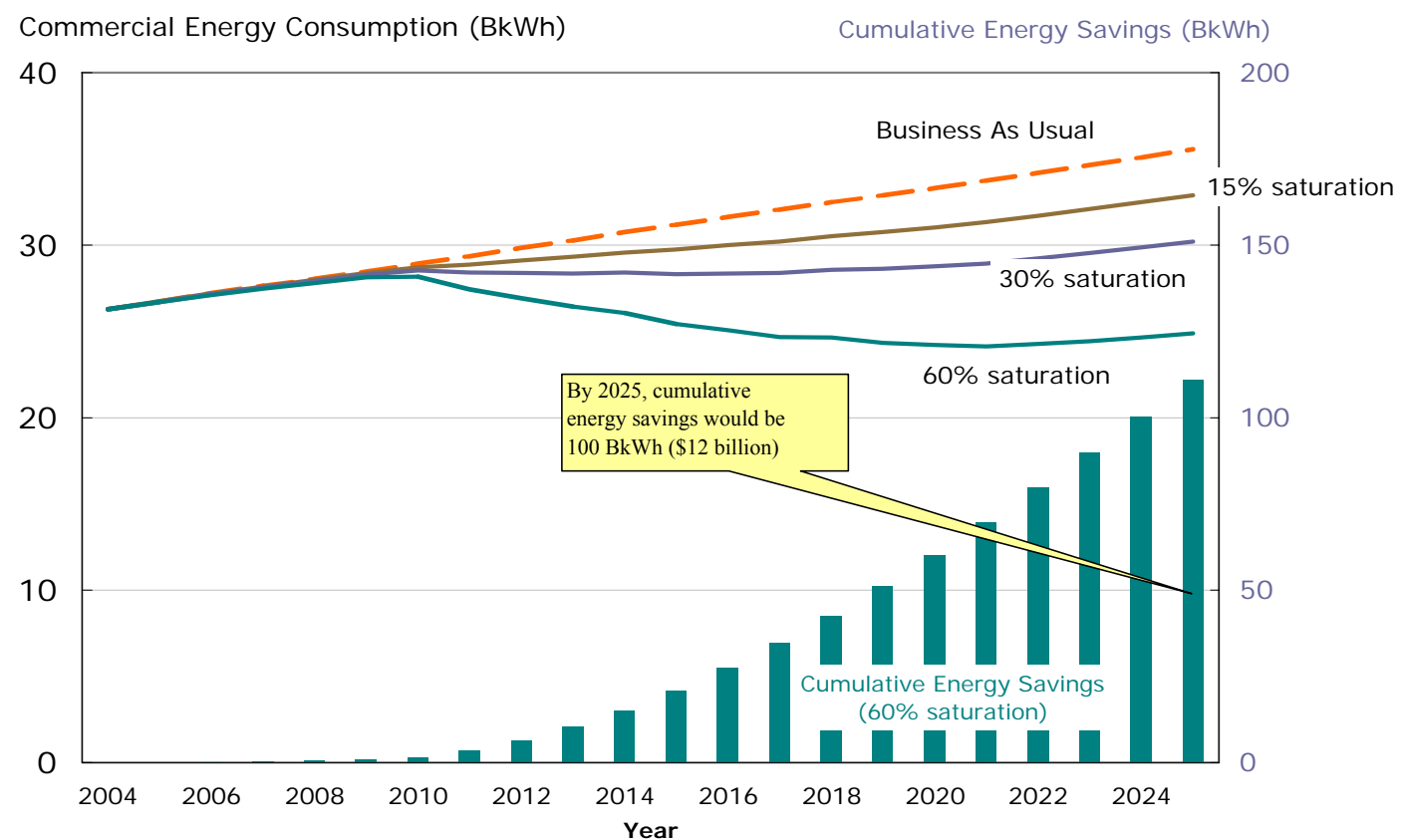

If advanced lighting controls were to achieve $60 \%$ penetration in the commercial building market in 20 years, the State would consume 100 Twh less electrical energy at a savings to ratepayers of $\$ 12$ billion. Over 20 years, it would reduce greenhouse emissions by 20 MMTCe -- the environmental equivalent of removing over 13 million cars from the roads. 


\subsection{Introduction}

\subsection{Background and Overview}

The goal of this study is to examine existing and emerging California commercial lighting technology, systems, and markets in terms of current and anticipated demand response programs. A specific objective of the study is to examine the electric peak demand related to lighting in commercial buildings, and develop a research plan to improve the demand response capability of such lighting. Another objective is to explore synergies between lighting technologies and systems that can provide both improved energy efficiency and demand responsiveness.

Electrical lighting in buildings is responsible for $30-33 \%$ of the commercial sector peak load [i]. As a result, lighting in commercial buildings should be considered as a potentially sheddable load during times of curtailment. HVAC and lighting system are the two largest contributors to the commercial electrical peak. Many demand reduction programs focus on HVAC since HVAC systems are connected to energy management systems that are relatively easy to connect to a demand response communications infrastructure. Lighting systems are less automated and are therefore more difficult to shed loads automatically. But lighting systems have one advantage over HVAC systems from a demand shed capability - a reduction in light level large enough to be electrically significant need not encroach on occupant comfort. If dimmable lighting were implemented across the entire commercial sector, the demand response potential of California building lighting systems would be enormous (see Figure 1below).
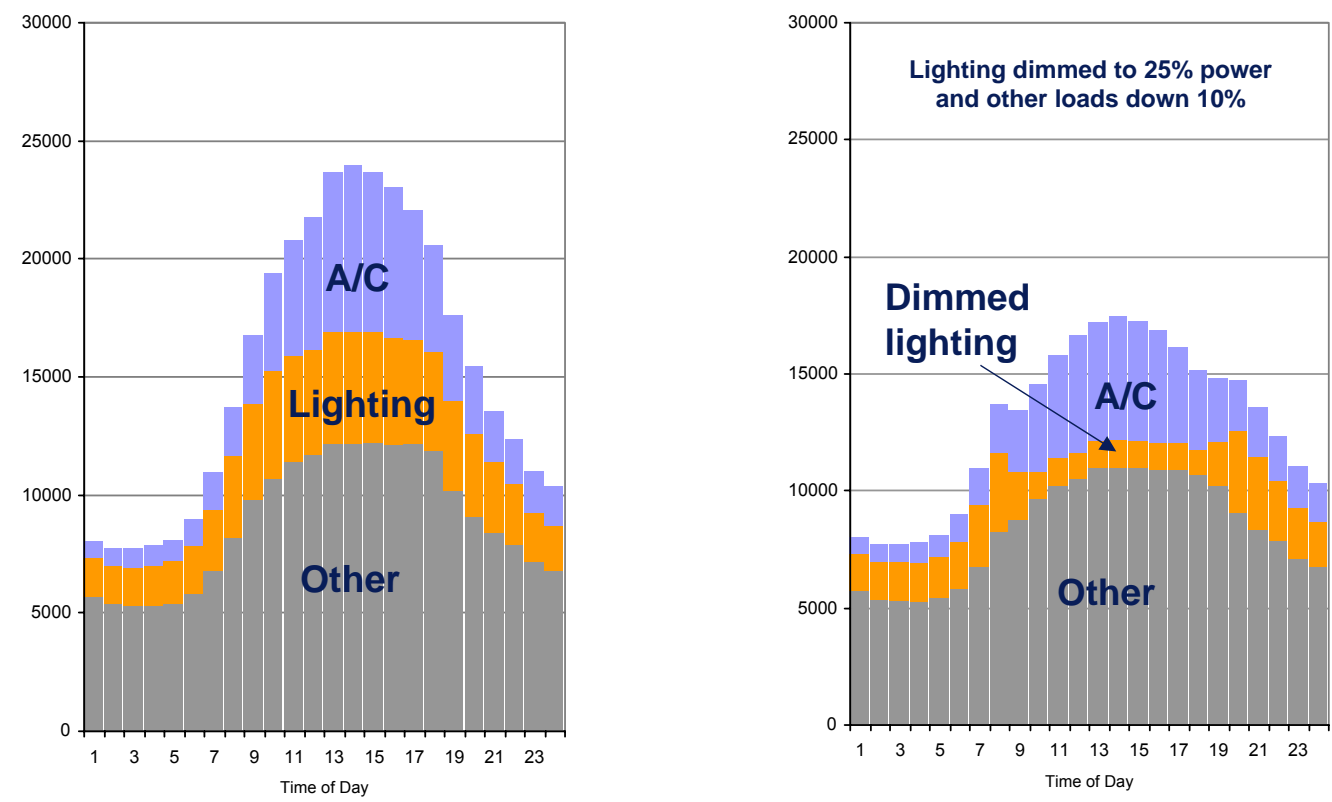

Figure 1: California commercial sector electrical demand (megawatts) for 1999 Summer Peak broken down by major end-use (left). Dimming all commercial lighting to $25 \%$ (right) could theoretically reduce total commercial peak by $7 \mathrm{GW}$.

This scoping study describes the load shedding potential of California building lighting systems and presents emerging dimming technologies and infrastructures that would greatly increase the shed potential of this $6 \mathrm{GW}$ sector. The study shows that the same technologies that increase load shed potential also double the effective energy efficiency of commercial lighting systems. 
Imagine unusually hot and humid weather conditions increasing the electricity demand beyond its forecasted levels. Or, imagine a tree falling and severing the transmission line and requiring redistribution of power through another transmission line, which may already be close to its maximum capacity. In each case the electricity grid operator needs to have reassurance that groups of customers can deliver the demand reduction required to keep the grid operational. Currently, demand response programs provide incentives and penalties for the program participants in order to provide the demand reduction when and where needed. However, simple factors such as the absence of the building operator on the day of a demand response event prohibit a building's participation in a demand response event. We envision a future where the grid operators can reduce lighting loads in commercial buildings with a push of a button in their service territory. This scoping study examines the potential in lighting systems in commercial buildings to participate in such demand reductions.

Demand response (DR) is a complex resource that requires communication and feedback between the supply side and demand side in order to be fully realized. In simplest terms, DR refers to the modification of customer electricity usage at peak times in order to help address system reliability and/or electricity market conditions and pricing. Currently DR programs are implemented with supply side notification and demand side response (open loop). Usually, supply side notification entails e-mail messages, text messages and/or telephone calls anywhere between a day ahead to a couple of hours ahead. Each building operator responds to the notification depending on the building use patterns during the event time and day, the level of discomfort that the event may cause and the financial incentives and penalties related with participation. We envision a future where the supply and demand of electricity is balanced through closed loop systems during a DR event where the aggregated demand reduction of participants is available to grid operators and/or utilities real-time.

Lighting is usually associated with visual health and safety. Any change in lighting has the potential to directly affect the safety and well being of the occupants not to mention their productivity. Therefore, commercial building operators are apprehensive about using lighting for DR. However, requirements for lighting depend on the task. Today's commercial buildings house many different tasks and technologies that enable the completion of these tasks. DR strategies for lighting systems in commercial builds have to consider all the issues related to the tasks of the occupants and the function of the building. In addition to the function, DR lighting strategies are also limited by the lighting infrastructure in the buildings. Zone size, zone distribution and the balance of local and central controls directly affect the function and cost of a lighting system and provide the frame for DR strategy development.

\subsection{Project Objectives}

This scoping study describes the load shedding potential of California building lighting systems and presents emerging dimming technologies and infrastructures that would greatly increase the shed potential of this $6 \mathrm{GW}$ sector. The study shows that the same technologies that increase load shed potential also double the effective energy efficiency of commercial lighting systems.

\subsection{Report Organization}

The report has seven sections. The first section describes a framework for demand responsive lighting for commercial buildings. The demand side management framework is discussed in the context of lighting controls and suggestions on various implementations are outlined. A summary of previous experience with demand responsive lighting systems follows. The next section characterizes lighting controls in commercial buildings in the U.S and in California and describes the lighting standards for commercial buildings in California in order to characterize lighting infrastructure in existing buildings. The fourth section identifies demand and energy consumption patterns of commercial buildings in California. The fifth part of the report describes the different 
technical pathways for upgrading the lighting in existing buildings. This section describes the different types of components, their inputs and outputs and communication protocols and technologies they utilize. Demonstration projects for DR lighting controls are matched with the pathways concept in the next section. The last two sections summarize the findings and present recommendations to deliver significant and reliable demand reduction in California's commercial buildings.

\subsection{Demand Response Issues}

\subsection{Demand-Side Management Framework}

Forecasting loads in advance is a major problem for electricity grid operators. Any prediction of demand response time and amount enables effective use of this resource. We envision a future where each building chooses lighting shed strategies, programs these into their lighting control system and when the price of electricity rises or there is a reliability concern, the demand relief is delivered within a certain response time and pre-specified amount. Lighting is one of the best targets for demand reduction because it can be delivered within a short period of time independently from external conditions (such as weather).

The demand-side management (DSM) framework presented in Table x provides three major areas for changing electric loads in buildings: energy efficiency (for steady state load optimization); peak load management (for daily operations); and demand response (DR) (for event driven dynamic peak load reduction). In this report, we present the DSM framework from lighting controls perspective and use load and demand interchangeably.

\begin{tabular}{|c|c|c|c|}
\hline & \multicolumn{3}{|c|}{ Demand Side Management } \\
\hline & $\begin{array}{l}\text { Efficiency and } \\
\text { Conservation } \\
\text { (Daily) }\end{array}$ & $\begin{array}{l}\text { Peak Load Management } \\
\text { (Daily) }\end{array}$ & $\begin{array}{l}\text { Demand Response } \\
\text { (Dynamic } \\
\text { Event Driven) }\end{array}$ \\
\hline Motivation & $\begin{array}{l}\text { - Utility Bill } \\
\text { Savings } \\
\text { - Environmental } \\
\text { Protection }\end{array}$ & $\begin{array}{l}\text { - TOU Savings } \\
\text { - Peak Demand Charges } \\
\text { - Grid Peak }\end{array}$ & $\begin{array}{l}\text { - Price } \\
\text { - Reliability } \\
\text { - Emergency }\end{array}$ \\
\hline Design & $\begin{array}{l}\text { - Efficient Shell, } \\
\text { Equipment \& } \\
\text { Systems }\end{array}$ & -Low Power Design & $\begin{array}{l}\text { - Dynamic Control } \\
\text { Capability }\end{array}$ \\
\hline Operations & $\begin{array}{l}\text { - Integrated System } \\
\text { Operations }\end{array}$ & $\begin{array}{l}\text { - Demand Limiting } \\
\text { - Demand Shifting }\end{array}$ & $\begin{array}{l}\text { - Demand Shedding } \\
\text { - Demand Shifting } \\
\text { - Demand Limiting }\end{array}$ \\
\hline
\end{tabular}

Table 1: Energy efficiency, daily load management and DR

Energy Efficiency: Energy efficiency objectives are usually met by lowering energy use while providing the same level of service. Driven by utility bill savings, the need to conserve natural resources and environmental stewardship, energy efficiency measures permanently reduce peak load by reducing overall consumption. In building lighting systems, improved energy efficiency has been partly achieved by installing energy efficient lamps and ballasts (Approximately $75 \%$ of the fluorescent lighting stock in California uses electronic ballasts. Nationally, the fraction is 
about $50 \%$ electronic and $50 \%$ magnetic ballasts). After more efficient equipment, lighting controls are the most effective method to reduce lighting energy consumption in commercial buildings. But the success of lighting controls in buildings has been mixed. Occupancy sensors, which improve overall lighting efficiency by switching off unnecessary lighting, are finding increasing use in commercial buildings. However, the benefit of occupancy sensors is largely during non-peak hours, making them less effective for peak demand management. Photo sensors for daylight driven dimming or switching have achieved much lower penetration rates because of system complexity, commissioning and cost issues. It is unfortunate that daylight-dimming systems have such low penetration rates in the commercial market since daylight following system effectively reduce peak demand in buildings at precisely those times when it is most expensive to produce energy.

Load Shapes for Load Reduciton Terminology

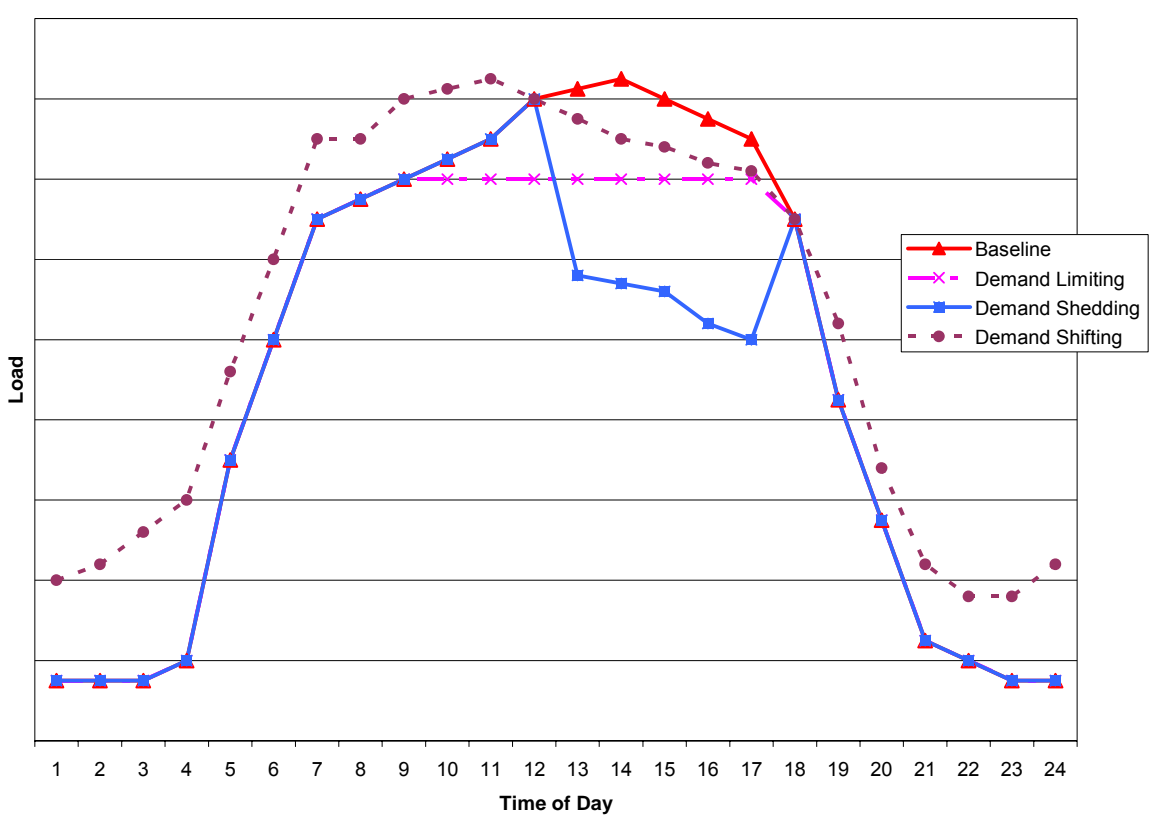

Figure 2: Demand profile of various demand response methods

Daily Peak Load Management: Advances in metering technologies have made it possible to differentiate electricity usage patterns between buildings. Peak load management is motivated by high charges for peak demand and time-of-use rates. Typical peak load management methods include demand limiting and demand shifting. Demand limiting refers to shedding loads when pre-determined peak demand limits are about to be exceeded. Loads are restored when the demand is sufficiently reduced. This is typically done to flatten the load shape when the predetermined peak is the monthly peak demand. Demand shifting is shifting the loads from peak times to off-peak periods. Figure 2 displays the typical demand profile of a commercial building employing these methods. Peak load management using lighting systems in buildings is not common practice. Changes in the lighting system tend to be visible and disruptive making daily peak management problematic. However, daylight driven dimming is used for daily peak load management in buildings where daylight is available and peak load is considered problematic. 
Demand Response: Demand response refers to the modification of customer electricity usage at peak times in order to help address system reliability, reflect market conditions and pricing, and support infrastructure optimization or deferral. Demand response programs may include dynamic pricing and tariffs, price-responsive demand bidding, contractually obligated and voluntary curtailment, and direct load control or equipment cycling. DR methods such as demand limiting and shifting can be utilized when the economics and reliability issues are predicted and communicated to each site in advance. Demand shedding is dynamic temporary reduction or curtailment of peak load when dispatched and refers to strategies that can be possibly implemented within a shorter period of response time. Lighting systems are good candidates for demand shedding because they have short response times and the demand savings are predictable and repeatable most of the time. The occupants tend to notice the change in lighting levels, especially when the change is abrupt but do not seem to be too inconvenienced if they know the reason.

There are two ways to strategize demand shedding with lighting systems in commercial buildings: absolute reduction and relative reduction. Absolute reduction is achieved by programming preset scenes for lighting levels in selected areas when demand response is dispatched. In the HVAC controls for DR, this is similar to setting the temperature up to a certain setpoint temperature. This may translate to the building lighting system in many different ways such as half the lights on, half of the lamps in a fixture on or $70 \%$ of full light output. The problem with this approach is that it does not yield any savings if the lighting system performance already matches the preset scene at the time demand response is dispatched. Therefore, although this approach is easy to implement with current lighting control systems, the shed amount is a variable depending on the building use and occupancy. Relative reduction is reducing loads with respect to their settings at the time demand response is dispatched. In the HVAC controls for DR, this is similar to changing the temperature setpoint by a certain $\Delta \mathrm{F}$. Instead of dimming to a preset value, a certain percent reduction over its current value is achieved during a demand response event. This strategy requires that the light output from the lamp or power output from the ballast is communicated back to the lighting control system and centrally closed loop control is required. Systems with such sophisticated controls tend to be newer and more expensive. The decision to implement absolute or relative lighting shedding depends on the building lighting infrastructure, the lighting use in the building and the capabilities of the installed lighting control system.

Five techniques for wiring and controlling lighting systems in commercial buildings are identified below.

1. Fixture switching (rows)

2. Fixture switching ("checkerboard")

3. Tandem wiring

4. Stepped Dimming

5. Continuous Dimming

These wiring methods and their implications for implementing demand response strategies in California buildings are discussed in detail in Section 2.

Figure 3 presents a selection process designed to help facilities managers determine whether or not demand response in worth implementing given the electrical wiring system and availability of lighting controls equipment in the building. A building operator can implement DR lighting strategies manually or semi-automatically. If central control of lighting systems is not available to the operator, the time and effort costs and savings and other benefits have to be computed for 
each facility. If central control of lighting is available to the operator, the next step is for him to understand the "granularity" of the lighting control, which is determined through a set of yes/no questions in Figure 3. Advanced lighting controls and increased levels of granularity allow us to define explicit steps in building lighting that can potentially be exercised during DR events.

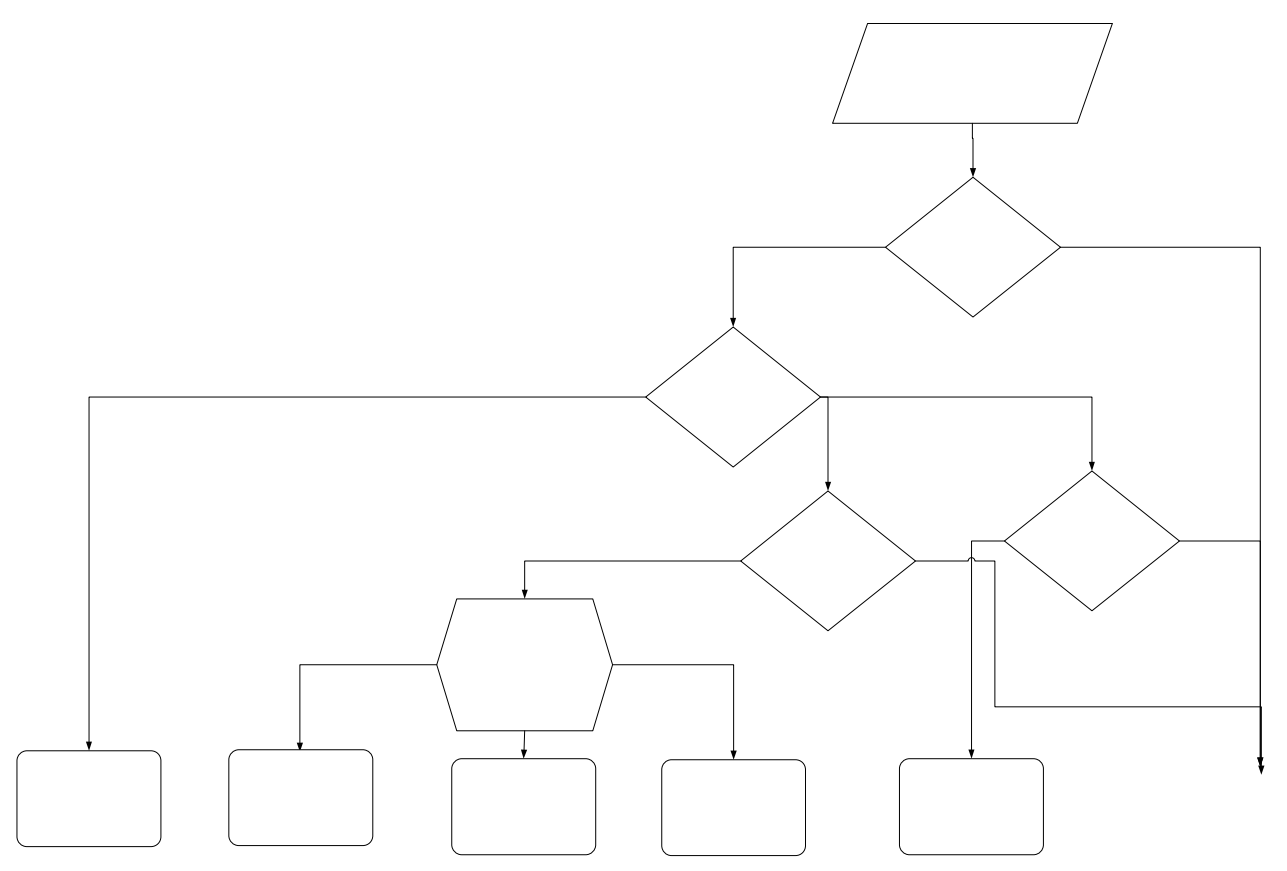

Figure 3: Flow diagram for DR Decisions for Lighting Systems

For retrofit applications, the ultimate choice of DR strategy is determined by the in-place building electrical system and the availability of appropriately-switched zones to implement DR and other energy efficiency strategies. It is important to stress that the choice of DR strategy: dimming, fixture switching, etc should not be separated from the choice of energy efficiency strategy. The size and location of lighting zones are important factors because as the size of the zone gets larger, it covers areas with varying occupancy, lighting requirements and daylight availability. Large zone switching strategies in particular may be objectionable to occupants in some applications and disrupt work flow.

The ease with which demand response can be implemented in existing buildings depends on the electrical wiring system, particularly the wiring for the overhead lighting. The practicality of switching overhead lighting depends on how the fixtures are circuited and lamp switching depends on how each lamp/ballast combination is wired.

\subsection{Previous Experience with Demand Responsive Lighting Systems}

This section describes prior historical experience with installing demand responsive lighting systems in real buildings.

\subsection{Load Shedding at the Watergate Office Building, Emeryville, CA}

[ii] describes the first documented case study of demand responsive lighting in the country. Light levels at this site were dimmed in response to a simulated load shedding signal of about one hour 
duration. As shown in the Figure 4 below, the shed reduced lighting power by about an additional $10 \%$ even though the lights were already significantly dimmed at that time because of daylight dimming. As shown in the figure, the impact on provided light level was small and was unnoticed at the time by building personnel even though they were aware of and were watching for the light reduction. This demonstration showed that a small change in dimmed light level is inconspicuous of accomplished with dimming and opposed to switching.
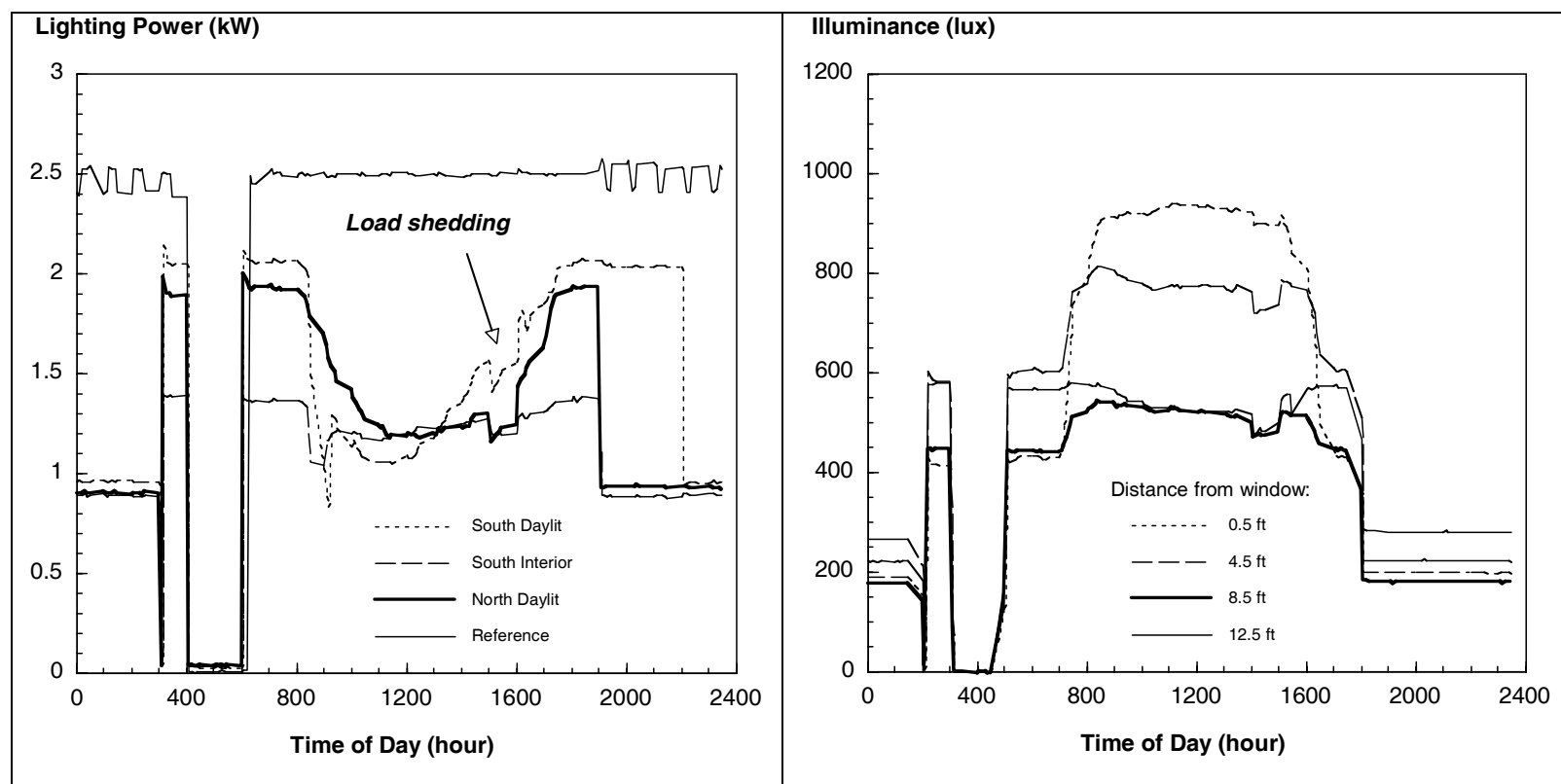

Figure 4. PGE-funded lighting controls research in 1991 was the first to examine the effectiveness of using dimmable lighting to achieve significant demand reduction (aka demand response).

\subsection{Implementing DR in a Supermarket}

[iii] describes how demand response lighting was implemented in a California supermarket which had an EMCS and zone switching. At this site, there was a central control system and furthermore, overhead lighting could be switched in rows ("row switching" above) simply by operating relays in the electrical closet. The in-place equipment (EMCS system) and appropriate electrical wiring allowed this site to respond effectively to a demand shed signal by switching off about $50 \%$ of the overhead lighting during the event. The impact of the tested demand shed strategies on building electrical demand is shown in Figure 5. The effect of the demand shed on lighting quality is captured in the "before" and "after" images shown in Figure 6. 

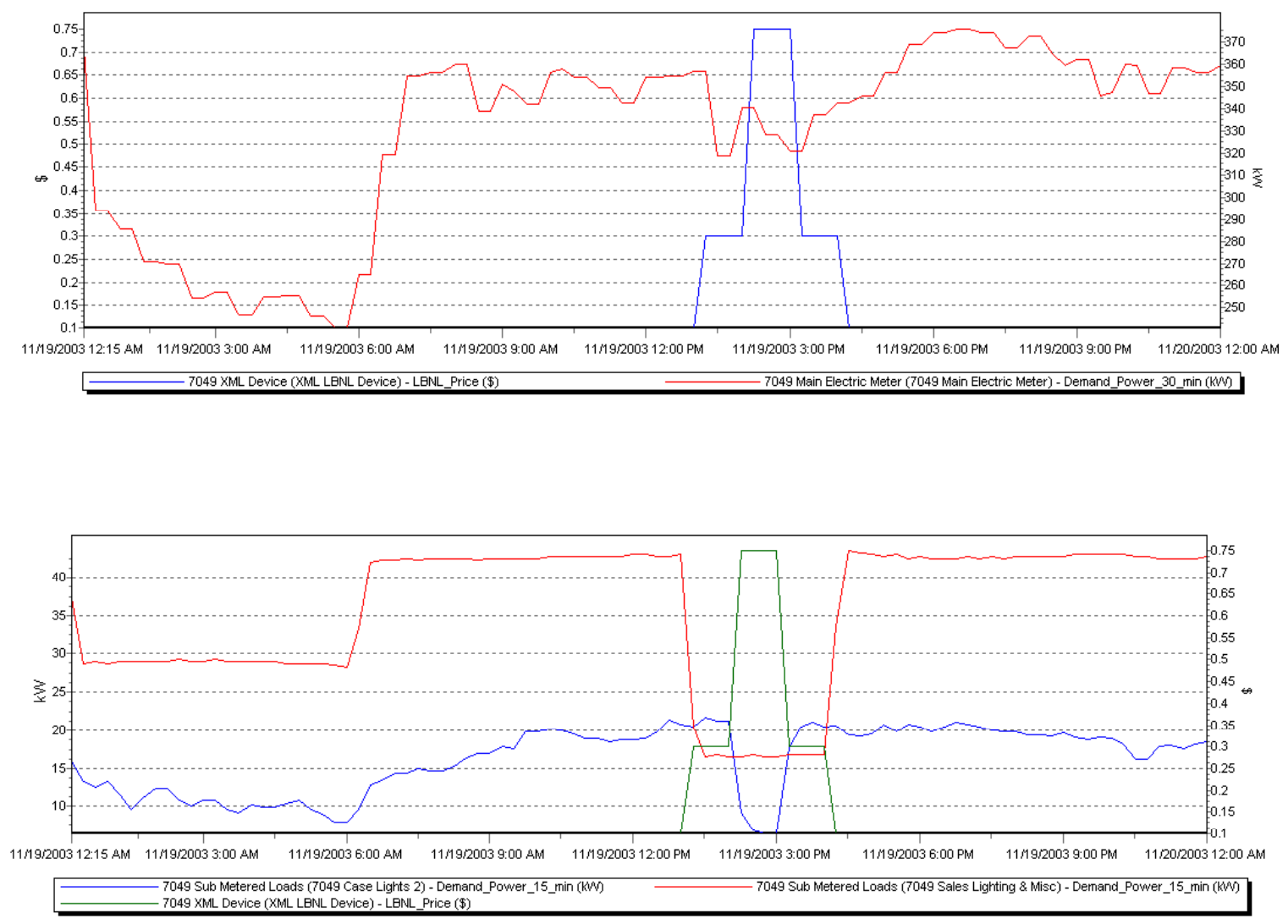

Figure 5: Total electric power in supermarket during two step load shed experiment.
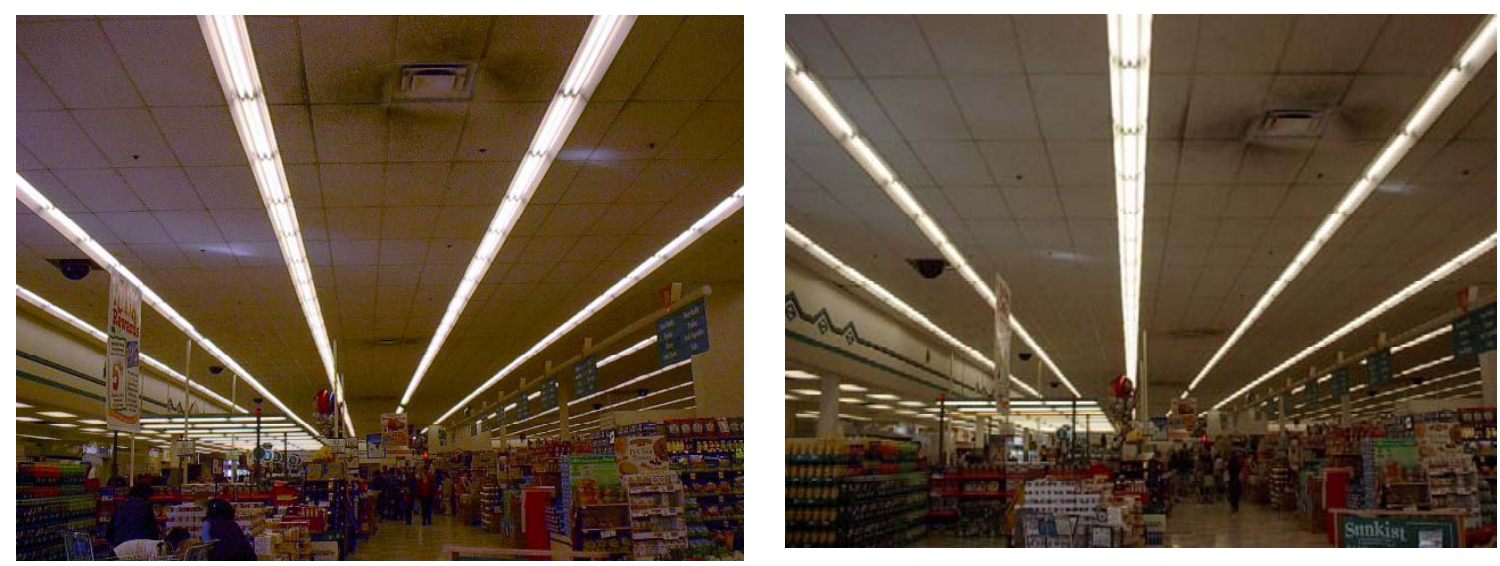

Figure 6: Overhead lighting at normal (left) and in load shed mode (right). In load shed mode, about $50 \%$ of the rows of lamps are switched off.

This illustrates the practical potential for demand shedding using switching in existing buildings. Obviously, there is a noticeable change when the rows of lights are switched off, possibly leading to occupant complaints. 
Levels of automation in DR are defined [iii] and are summarized as follows:

Manual Demand Response involves a labor-intensive approach such as manually turning off or changing comfort set points at each equipment switch or controller.

Semi-Automated Demand Response involves pre-programmed load shedding strategies initiated by a person via centralized control system.

Fully-Automated Demand Response does not involve human intervention, but is initiated at a home, building, or facility through receipt of an external communications signal.

Most DR activities involving lighting systems fall in the manual DR category and very little in semi-automated category. This stems from the fact that advanced lighting controls are not widely deployed in the U.S [iv]. Only 3\% of commercial buildings have lighting controls making up 7\% of commercial floor space.

Although the lack of advanced lighting controls that allow for centralized control of lights in commercial buildings is a barrier, this section only presents technically feasible vision of fully automated DR for lighting systems. The DRRC automated DR studies from 2003, 2004 and 2005 show that fully automated DR for lighting using existing building infrastructures is technically feasible and produce repeatable results. Two chains (Albertsons and Target), both designed with switching lighting systems as a Title 24 requirement, and a building controls company (Echelon) with dimmable lighting were participants in the automated DR studies. The maximum electric peak demand intensity savings for each facility is summarized in Figure 7. All of the sites had centrally controllable lighting systems. The authors consider Albertsons and Target to be potentially high impact customers because of the similarity of each of their buildings, lighting system infrastructures and central energy management approach. Their corporate energy management groups are located outside of California. They were able to receive a communications signal indicating a change in electric price and control the associated store remotely. Customers' reaction to reduced light levels is a concern for both chains especially during the high traffic times.

Beyond the technical feasibility of remotely controlling lights, the coordination of each store with its corresponding utility and DR program for a state and communication with a central energy management group can introduce a complex problem that is not addressed in this report. 


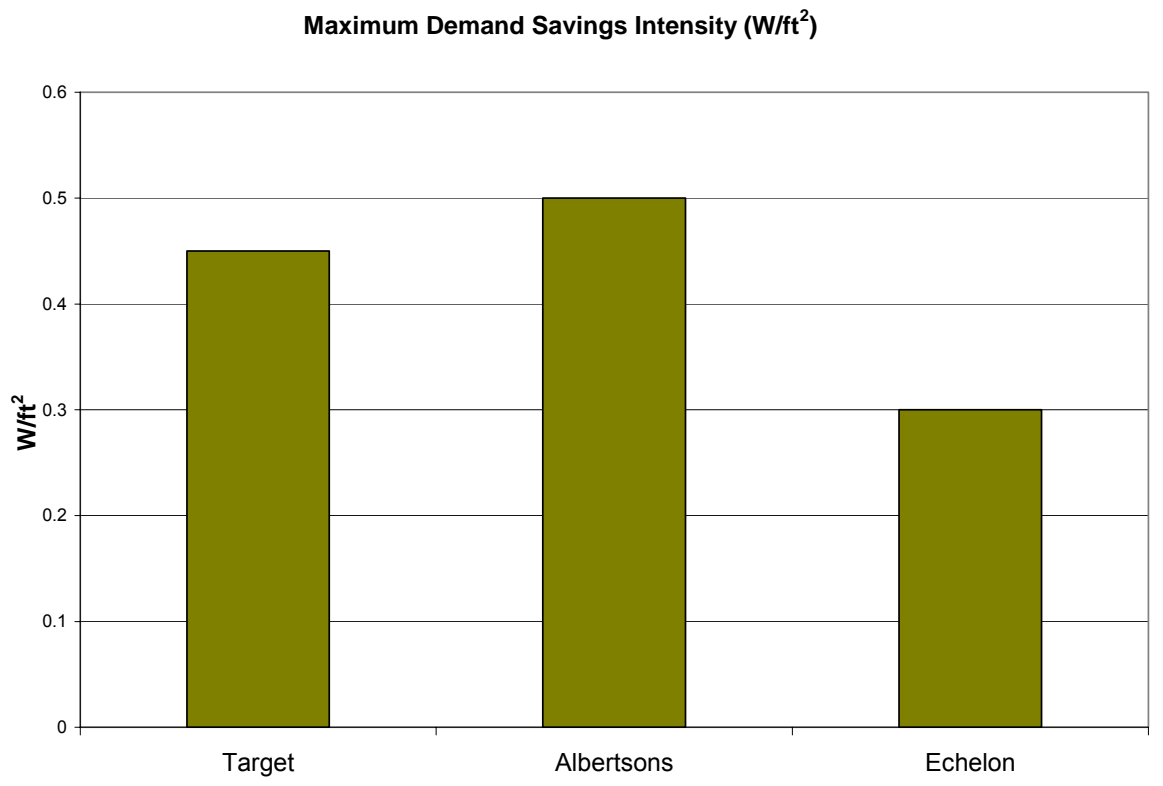

Figure 7: Maximum Demands Savings Intensity due to Lighting System Response

\subsection{Demand and Energy Consumption Patterns of California Commercial Buildings}

\subsection{Demand}

Lighting demand is particularly significant during hot, summer days. As shown in Figure 8, lighting is the single largest end-use in CA commercial buildings for the 2003 peak day (31\%), even higher than air-conditioning [ $\left[^{\mathrm{V}}\right]$. For the 3 hour peak (2:00 PM - 5:00 PM), lighting is 30\% of the load, while $\mathrm{A} / \mathrm{C}$ is only slightly larger (32\%). 

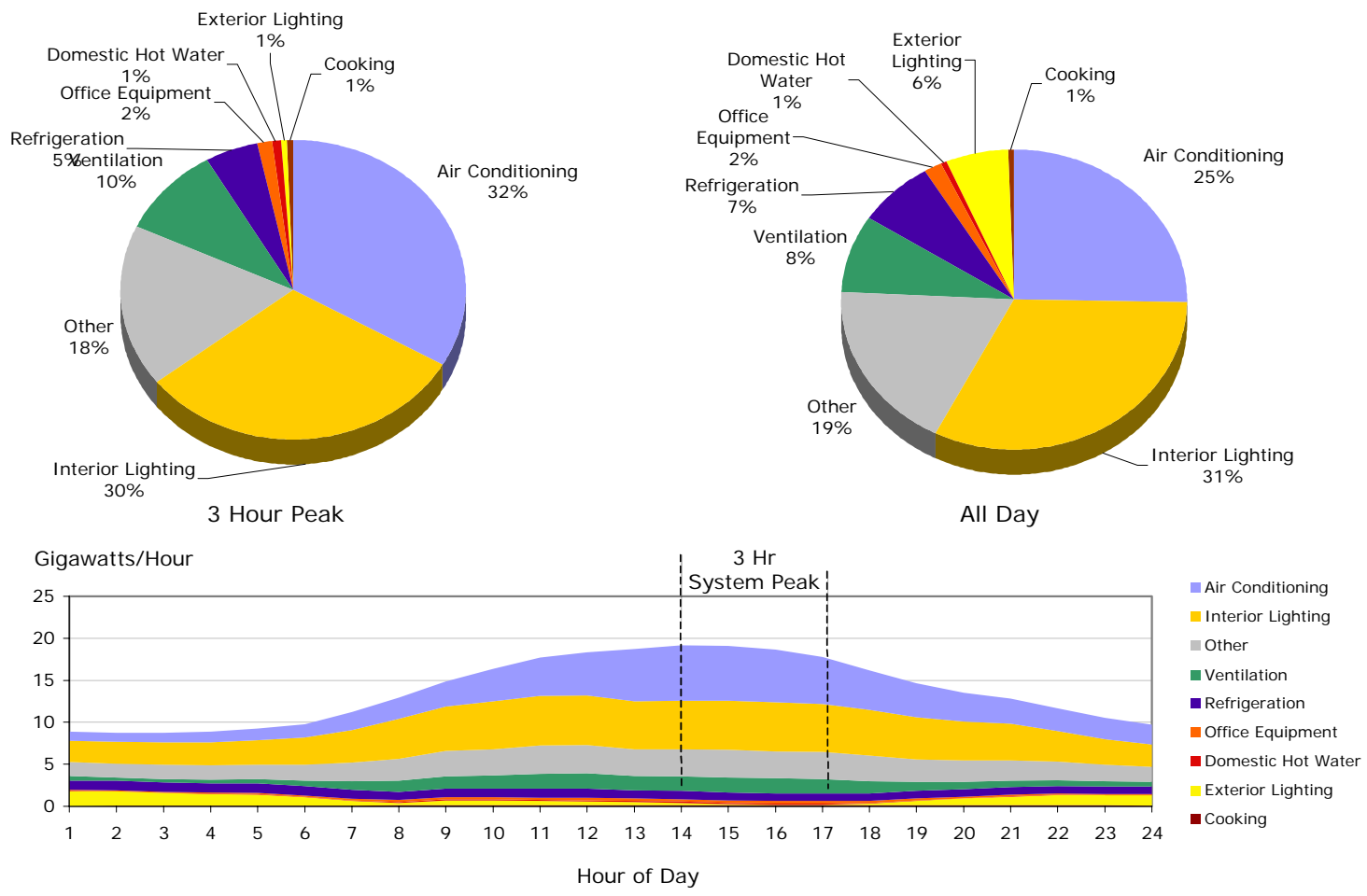

Figure 8: End Use Loads for California Commercial Sector on 2003 Peak Day [v]

The peak demand contribution from lighting in commercial buildings on a peak day in 2003 in California was nearly $6 \mathrm{GW}$. A $20 \%$ goal of peak demand reduction has the potential to yield nearly $1 \mathrm{GW}$ of peak demand. This scoping study examines at the infrastructures, technologies and implementations that allow for tapping into the potential demand savings in commercial buildings.

\subsection{Energy}

The California Energy Commission has analyzed lighting energy usage across different building types in the State [v]. These data are shown in Figure 9. 


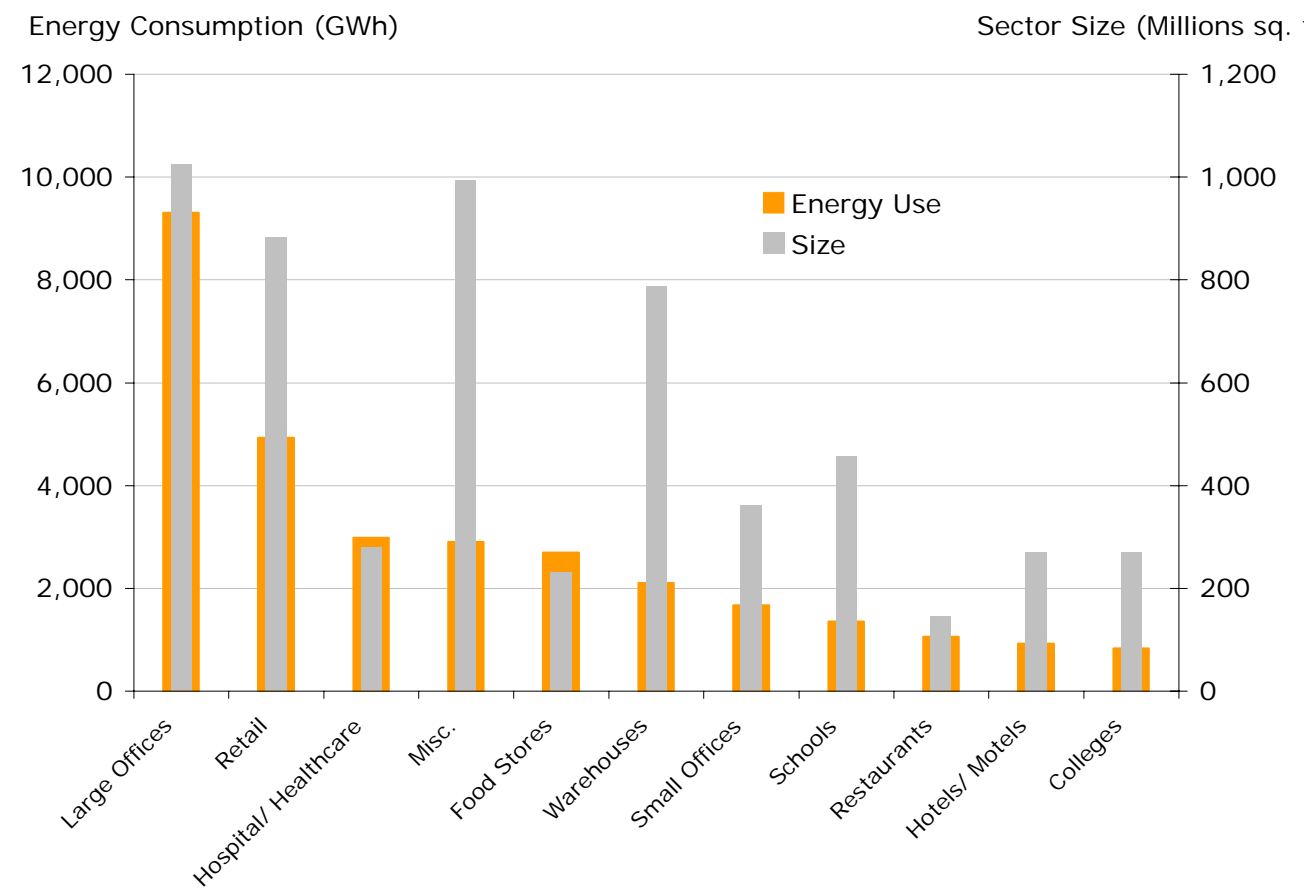

Figure 9: Lighting Energy Usage for Commercial Building Lighting in California 2000

California office buildings are the largest lighting energy consumer, followed by retail, hospital, miscellaneous and food stores. These five market sectors constitute nearly $75 \%$ of the $30.4 \mathrm{GWh}$ of lighting energy consumed in all California commercial buildings. Large offices, retail, hospital, miscellaneous and food stores make up nearly $60 \%$ of the 5.9 billion sq. $\mathrm{ft}$. of commercial floor space. This market data indicates that it is appropriate to focus energy (and demand) reduction programs on these major market sectors.

Other data from the same source provides a breakdown of California commercial lighting energy by all major light source types. Of the 30,739 Gwh of lighting energy consumed in California's indoor commercial sector, $85 \%$ of the energy is consumed by fluorescent lighting systems. Thus fluorescent lighting is clearly the dominant light source with most sectors using 4 -foot and 8-foot fluorescent lamps. 


\section{Commercial Indoor Lighting Energy Use}

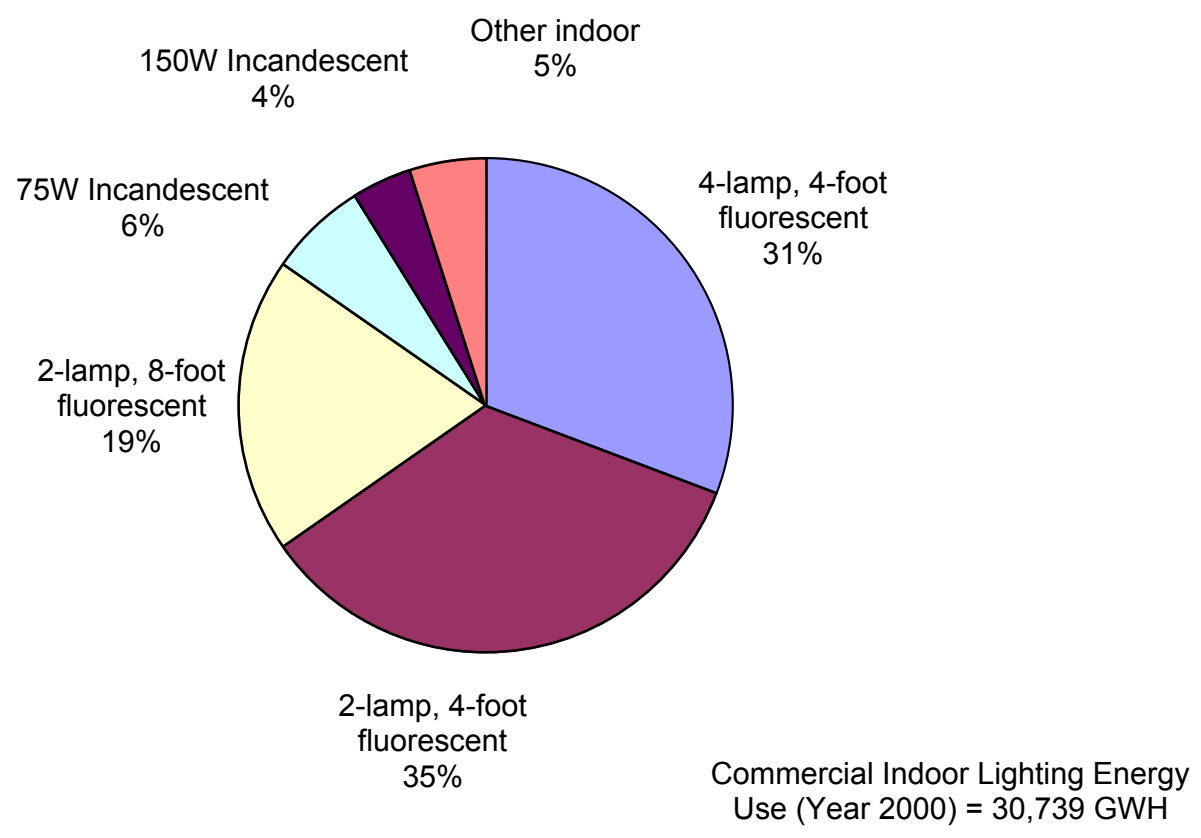

Figure 10: Commercial Indoor Lighting Energy Use

\subsection{Prevalence of Lighting Controls}

Another important attribute of the California commercial building stock is the prevalence (or absence) of lighting controls in this market segment. According to the Commercial Buildings Energy Consumption Survey [iv] conducted in 2003 and recently published results online, lighting controls is not common practice in the U.S. 3\% of commercial buildings have lighting controls making up 7\% of commercial floor space. By contrast, $7 \%$ of commercial buildings making up $31 \%$ of commercial floor space have energy management and control system (EMCS) for HVAC system. 


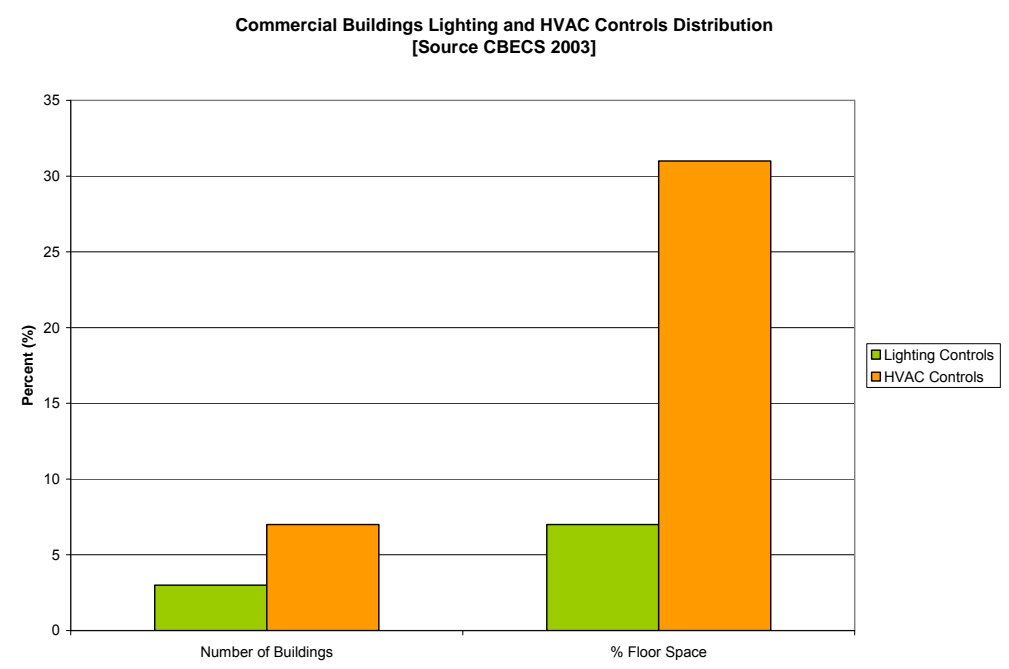

Figure 11: Lighting Controls Penetration Compared with HVAC Controls in Commercial Buildings

While the absence of lighting controls nationally is discouraging, the penetration of lighting controls into California buildings is expected to be higher due to California's Building Standards (Title 24 and 20) as well the greater awareness of the lighting controls for energy efficiency and the research and policy support that has been provided by the CEC and the utilities.

\subsection{Impact of Title 24 on Lighting Systems}

The Energy Efficiency Standards for Residential and Nonresidential Buildings were established in 1978 in response to a legislative mandate to reduce California's energy consumption. Over the years, these standards, also known as Title 24, have played an important role on the design of infrastructure of major building systems. These standards are updated periodically to allow consideration and possible incorporation of new energy efficiency technologies and methods.

California's Title 24 has had major positive benefits on the electrical wiring infrastructure of California commercial buildings. Because Title 24 has required "bi-level" lighting since 1983, much of California's existing stock includes improved lighting switching [ ${ }^{\mathrm{vi}}$. Title 24 requires multiple lighting level controls in controlled spaces of a certain size. Typically, with bi-level switching, office occupants are provided with two wall switches near the doorway to control lights. In a typical installation, one switch would control $1 / 3$ of the fluorescent lamps in the ceiling lighting system, while the other switch would control the remaining $2 / 3$ of the lamps. This allows four possible light levels: OFF, 1/3, 2/3 and FULL lighting.

While the Standard requires bi-level switching for many applications, it does not state the exact method for implementing it. Five common ways of implementing bi-level lighting are given below:

- Fixture switching (rows): Separately switching "on" alternative rows of fixtures. When fixtures are wired in rows, fixture switching is implemented by switching half the rows of fixtures from one switch and the remaining rows with a second switch. This is commonly done in supermarkets (alternative row switching) and in schools with pendant-mounted 
fixtures in rows. Fixture switching done in rows runs the risk of increasing lighting nonuniformity.

- Fixture switching ("checkerboard"): Separately switching "on" patterns of fixtures. To switch fixtures in patterns, every other fixture in the space is wired to one switch and the remaining ones to a second switch (also called "checkerboard" switching). "Checkerboard" switching provides more uniform light levels than fixture switching done in rows and is used in supermarkets and big box retail.

- Tandem wiring: With tandem wiring, multi-lamped ballasts are wiring so that some lamps in each fixture are wired to one switch and the remaining to a second switch. With three-lamp luminaires, the "inboard" lamps are one switch while the "outboard" lamps are on a second switch. Three-lamp luminaires tandem wired provide 3 levels of light: $1 / 3,2 / 3$ or full lighting. When tandem wiring is used for two- or four-lamp fixtures, only two distinct light levels are available: $1 / 2$ or full lighting. This wiring method is often used in offices, educational, and healthcare buildings.

- Stepped Dimming: With stepped dimming, all the lamps in a fixture are controlled together to provide one of two (or three) light levels within a space. This requires stepped dimming ballasts, which have two hot leads (A and B) rather than one. All the A leads are tied to one switch and the B leads to the second switch. Bi-level and tri-level stepped dimming ballasts are available from several ballast manufacturers, but they are considerably more expansive than standard ballasts. A description of stepped dimming ballasts is presented in Section 5

- Continuous dimming: With continuous dimming, the lighting in the overhead fixtures can be continuously varied from maximum light output to about 10\% light output. A detailed description of dimming ballasts is presented in Section 5. Because dimming ballasts are currently expensive, their use is generally restricted to high-end conference rooms in Class A offices. Some big box retail stores are increasingly using continuous dimming in conjunction with skylighting.

Although the standard does not specify centralized controls and most of the dimming controls are done by local photo-sensors and control hardware integrated at the ballast level, the existence of dimming ballasts and division of circuits or separations of ballasts does lay the foundation for manual and with some technical augmentations semi-automatic demand response.

\subsection{Analysis}

Because of Title 24, California has been able to maintain per capita electricity usage at the same level for the past 30 years while most other States have increased theirs. What is less well-known is that Title 24 has had long-term beneficial effects on the building lighting wiring infrastructure. Because the Standard requires that new buildings be wired so that multiple light levels are provided for each space, building occupants have a greater degree of control over their lighting than other states. It is easy for building occupants to obtain more than one light level in a space when they naturally take advantage of this control flexibility. Fortunately, energy savings is the usual result of people taking advantage of this switching infrastructure. The use of improved switching by building occupants saves the state some $\$ 100-150$ million annually in avoided energy costs [calculated from [vii]].

Gedunken Experiment - Imagine a heat wave where California's electrical infrastructure is strained to the breaking point. Imagine there is capability to trigger the bi-level switches in all California's buildings from HIGH to MEDIUM (or LOW) in one step. How much demand response is available?

The calculation- As mentioned, offices, schools, retail stores and foodstores consume about 15 $\mathrm{BkWh}$ is used each year in California for lighting offices, schools, retail stores and foodstores. 
Assuming an average 3200 hours a year operation, this equates to 4.7 Gigawatts average. Assume that bi-level switching only affects $75 \%$ of this load $(3.5 \mathrm{GW})$. Assume that $20 \%$ of the remaining load has already been taking advantage of the low switch level and so is excluded from further reductions. This leaves $2.5 \mathrm{GW}$ of demand response potential. If the lever were thrown, this 2.5 GW load would be reduced by about one half (remember that going from HIGH to LOW reduces the power by two). Thus the State could save $1-1.25 \mathrm{GW}$ just by turning everyone's bi-level lighting from full to medium!

In the following section, we describe technologies that could harvest this potential.

\subsection{Lighting Control Pathways}

In this section, we present a generic framework for a lighting controls architecture that could be implemented throughout the State to provide significant energy savings and full demand response in California commercial buildings. The architecture consists of four stages: input, user interface, control and communication and output. The architecture is intentionally generic; i.e., manufacturer- and network-neutral. Most lighting control companies and ballast companies offer a wide range of components that support one or more of the framework stages.

After detailing each stage below, we present examples of specific pathways for implementing lighting controls in buildings. Each pathway is a set of specific lighting control components providing end-to-end control all the way from the input to output stage using components that are selected to be compatible and designed to interoperate properly when organized as a system. We propose a generic lighting control pathway (Figure 12), then describe each generic piece more detail. This section concentrates on dimmable ballasts and their role on demand responsive lighting.

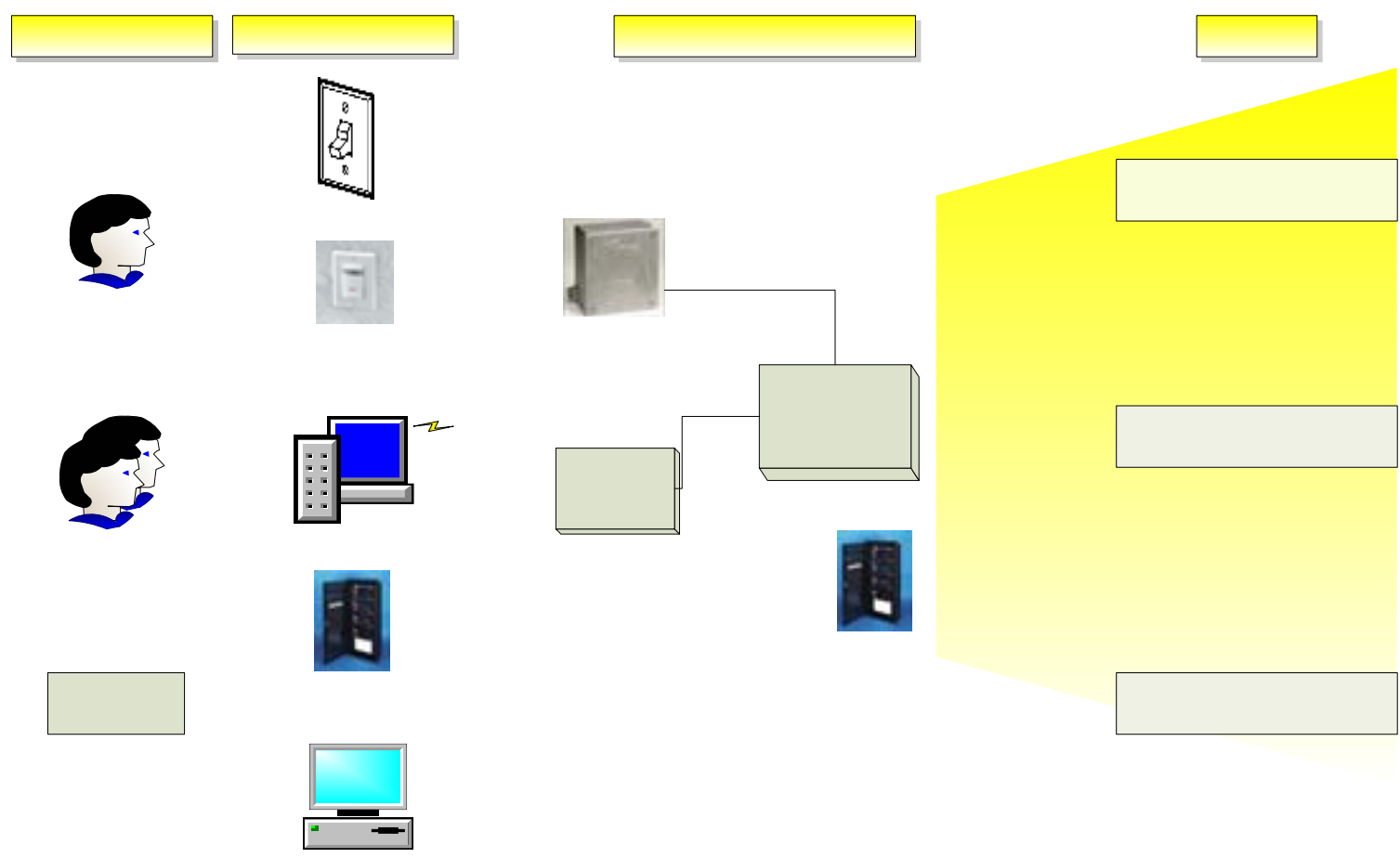

Figure 12: A Framework for Lighting Control 


\subsection{Input}

The input to any lighting control system can be divided into two categories: inputs from people and inputs from devices (usually sensors). The first category of input is from people causing a change in the system due to comfort or conservation concerns.

The second category of input is a sensor, which detects changes in the luminous environment. These sensors are typically light sensors that measure either illuminance or luminance within the controlled space. The light-sensing device in a photo sensor is typically a photodiode, a photoresistive cell or a photo-transistor. These devices respond to infrared, ultraviolet and/or visible light. Photosensors are designed with circuitry to respond to visible light. A photosensor is characterized by its spatial response and spectral response. Spatial response is the sensitivity of the photosensor to light shining in from various directions. Spectral response is its sensitivity to visible light with varying wavelengths.

Using photosensors to control interior lighting is not trivial and requires proper design, placement and calibration and commissioning. Illuminance sensors have a wide field of view and are recommended to be placed on or close to the work surface. Luminance sensors have narrower field of view. These sensors are usually placed on the ceiling oriented to "see" the work surface. There are two ways to adjust the gain of a photosensor. If the signal detected by the sensor is weak, it can be amplified with electronic circuitry or placing the sensor closer to the light source.

The output signal of a photosensor can control lighting one of two ways: 1) by turning the lights on or off depending on the set threshold; or 2) by dimming lights depending on the dimming response function identified during commissioning. It is important to note that for a $0-10 \mathrm{~V}$ dimming ballast, dimming does not occur through the entire range and it is typically limited to 2$8 \mathrm{~V}$ range.

\subsubsection{User interface}

Whether the input is a person or a sensor, there must be some user interface between the input and the lighting system. This interface usually takes the form of a switch that can be manually activated by the occupants, an automatic switch (such as an occupancy sensor) a remote control, a lighting panelboard or a computer. Wall switches are the primary method today for occupants to control their lighting systems so these controls should be accessible and their function clear. A person controlling the lights in his or her workspace may have a switch, an occupancy sensor and sometimes even a remote control device available to interact with the lighting control system. Often only authorized maintenance personnel have access to lighting panelboards or lighting control computer. A person controlling the lights in an entire facility may have access to a computer or a lighting panel to make changes in the lighting system. This section defines some of the devices that allow users to act to make changes to the lighting system.

\subsubsection{Wall switch}

A wall switch is the oldest and simplest way for manual control of lights. It directly controls the power to a fixture or to a set of fixtures. In offices, usually one wall switch is allocated per zone. Since 1978, in buildings that comply with California Title 24, two switches per zone are usually deployed.

\subsubsection{Occupancy sensor}

There are three types of sensing technologies utilized in occupancy sensors: passive infrared (PIR), ultrasonic or acoustics. Dual technology products use a combination of acoustic or ultrasonic with passive infrared sensing technologies. PIR sensors respond to movements of an infrared source, i.e., human body. The response is most sensitive to vertical movements and least sensitive to movements directly to and away from the sensor. The sensitivity of the sensor 
depends on the patterned lens being used to concentrate signals on the detector. Ultrasonic occupancy sensors emit ultrasonic waves between 25 to $40 \mathrm{kHz}$ at levels over $90 \mathrm{~dB}$. Motion is detected when the sound waves hit a moving object and cause a Doppler shift in the frequency. Acoustic sensors respond to sound pressure levels in the space and typically used with PIR sensors to reduce the occurrence of false offs.

Sensitivity adjustments such as signal strength, time clocks, and simple logic for occupant sensors are usually available to adjust the operation for specific room conditions. The input from the detector is adjusted and translated into a binary output that indicates occupied or unoccupied. This signal directly controls a relay to switch the lights on or off and usually incorporated in the sensor device. Besides turning lights on or off, the output of the occupancy sensors can be used for bi-level switching. In the future, the information from the occupant sensors is envisioned to be utilized for emergency evacuations, or adjusting HVAC set points in zones with no occupancy.

\subsubsection{Remote control device}

Remote control devices deliver access to the wall switch to the occupant's location. A remote control is a transmitter with either programmable or pre-programmed buttons that control the power to a fixture or to a set of fixtures. It allows the occupant to turn the lights on, off or dim. It communicates with a transceiver located in the ceiling plenum or directly at the fixture. The signals are transmitted at varying frequencies depending upon the interference issues related with equipment located at each facility. Communications using infrared or radio frequency are the most commonly used media for remote lighting controls.

\subsubsection{Computer interface}

A computer interface is provided to the building operator when a lighting control system is installed. In most installations, the software provided to the building operator facilitates the creation of schedules for weekdays, weeknights, weekends and regular maintenance. More sophisticated lighting control systems provide software tools that allow the operators to commission the lighting and conduct continuous maintenance as well as allowing regrouping of lighting fixtures to change existing lighting zones in order to meet organizational flexibility requirements of a facility.

\subsubsection{Lighting Panelboard}

Lighting panelboards are equipped with circuit breakers with capacity to handle the designated load. In their most basic form, facility operators can turn off entire circuits off with these panels. The advance of micro-processor based controllers allowed the panelboard designers to combine circuit breakers with micro-controllers to create lighting control panels. Advanced lighting controls panels:

- Communicate via building systems communication protocols such as BACnet or Modbus

- Include electronic clocks that allow for schedule creating.

- Group branch breakers to be controlled by schedules, manual inputs or override commands

- Monitor and log power consumption and demand

- Enable operators to set load shed levels on each breaker

- Generate alarm logs and trend status changes.

\subsection{Control and communications}

In the previous section, it was noted that lighting systems are operated with switches, relays and, sometimes, circuits of dimmable lighting. Lighting controls and any associated communications scheme constitute the third stage of the lighting control framework. Functionally, the lighting 
control system implements a particular communications protocol (either analog or digital) to dim and/or switch groups of downstream lighting fixtures (the fourth stage).

The specific choice of protocol is largely driven by the choice of lighting equipment to be controlled, especially the type of ballast. (A detailed discussion of the different ballast types is presented in the next section).

There are many types of lighting control systems available today from well-established companies, but generally these controls are installed during building construction. Because most lighting controls require additional wiring, they are often cost-prohibitive to install in existing buildings.

The focus of the present scoping study is existing buildings where conventional lighting controls are generally not considered. Over the last few years, several companies are producing control hardware that can be installed into existing building electrical distribution systems. For example, General Electric now makes a product which is an application specific controller (ASC) intended to both switch loads and dim a 0-10 VDC dimming ballast circuit. Unlike conventional control hardware, manufacturers are designing and packaging the controllers to be installed onto existing electrical junction boxes.

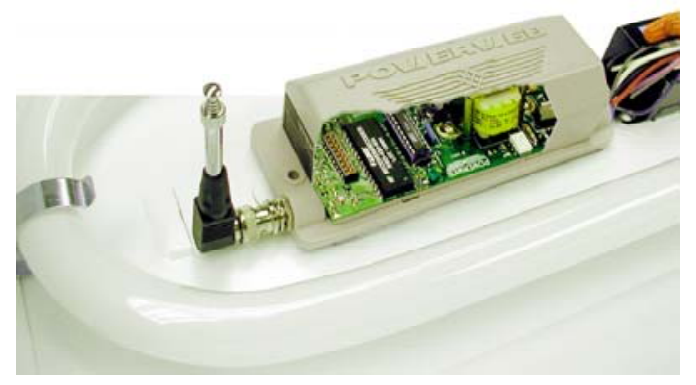

Figure 13. Application-specific controller produced by Power Web Technologies. The addressable controller can control one relay and two 0-10 VDC analog dimming ballast loops. A wireless network communicates with the controller through the RF antenna shown at the left.

Furthermore, some of these emerging controllers are equipped with radio-frequency inputs allowing them to be controlled from a wireless network. This makes these controllers ideally suited for retrofitting into California buildings in order to deploy advanced lighting control strategies into today's building stock.

Some different configurations for applying controllers to existing buildings are presented in Table 2.

- No ballast change-out required.
- Single switch leg is controllable.
- Commercial system available.
- Lighting loads, from 3 to 200
amps




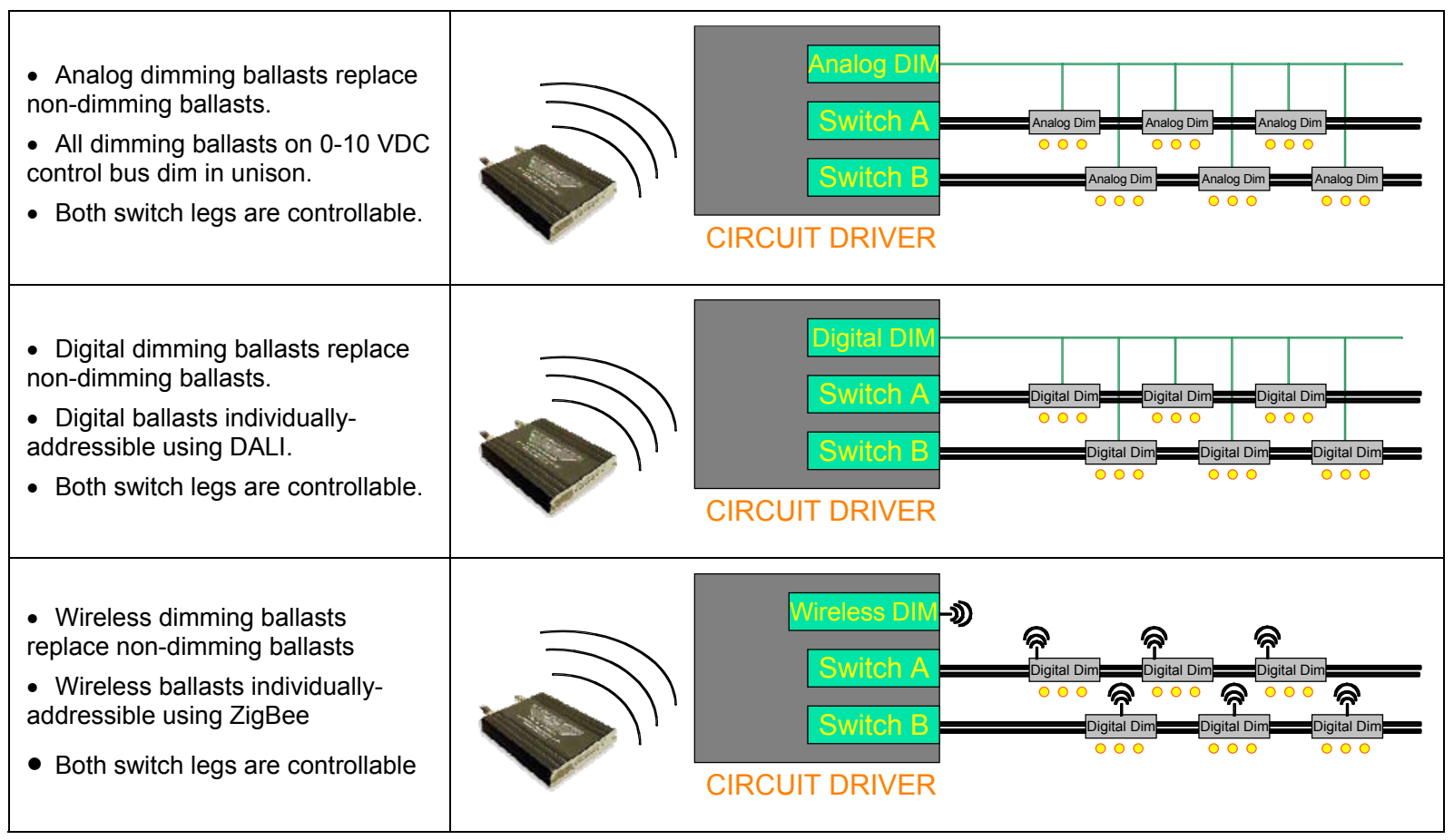

Table 2. Different configurations for applying controllers to existing buildings

The performance of controllers from two different companies is given in Section 5.3.

\subsubsection{Ballast type}

All fluorescent lighting systems use ballasts for operating fluorescent lamps. Ballasts that can operate connected lamps at different light output levels are useful for implementing demand response and many energy efficiency strategies. The ability to vary light level within a given area provides a flexibility that would be absent if only one light level was possible.

Not all dimming ballasts can be dimmed continuously. Some ballast manufacturers produce multi-level ballasts that operate lamps to one of several light levels. Below, both types of ballasts are discussed.

\subsubsection{Stepped dimming}

Stepped dimming (or multi-level) ballasts operate lamps to one of several discrete light levels. Tri-level $(33 \%, 66 \%, 100 \%)$ and bi-level $(50 \%$ and $100 \%)$ ballasts are available and are typically controlled by the additional of a second hot wire to the ballast. By selectively switching the black wire, the red wire or both, various combinations of light output are provided. One manufacturer offers multi-level ballast with asymmetric light levels (typically full and a low, standby level). Asymmetric multi-level ballasts are useful for applications, such as building stairwells, where a low-standby level of light is required even when the space is unoccupied.

Because of California's bi-level switching infrastructure, stepped dimming ballasts can be used to upgrade bi-level switched lighting systems without rewiring. Existing ballasts can be replaced with stepped dimming ballasts and one light switch tied to all the ballast black wires and the second light switch to the ballast red wires. Thus with only a ballast change-out, a bi-level lighting system can be upgraded.

Unfortunately, there is currently not a large market for stepped dimming ballasts and they are therefore similar or only lightly lower in price than continuous dimming ballasts. 


\subsubsection{Introduction to dimming ballasts}

This section of the scoping study discusses modern dimming ballasts. We briefly describe the different types of commercially available dimming ballasts, including both analog and digital ballast types. The key technical attributes of dimming ballasts from the standpoint of understanding their relative energy performance over the entire dimming range are presented in Appendix A.

\section{Major Types of Dimming Ballasts}

There are several different types of dimming ballast on the market today. (For a more complete discussion of dimming ballasts, see [ $\left.{ }^{\text {viii }}\right]$ ). There are two broad categories of ballasts - analog and digital. Dimming ballasts that are controllable using a 0-10 VDC control loop are the most common and have been available for many years. Approximately one million dimming ballasts are sold each year in the US compared to the approximately 60 million unit market for fluorescent ballasts as a whole. Digital ballasts are relatively new and probably constitute no more than 100,000 units in 2005.

\section{Analog dimming ballasts}

Analog dimming ballasts have two extra leads (violet and grey wires), which form a low voltage control circuit ("bus"), operates all connected ballasts to the same dimming level (intensity). Up to about 50 ballasts can be wired in parallel to the control bus as shown in Figure 14.

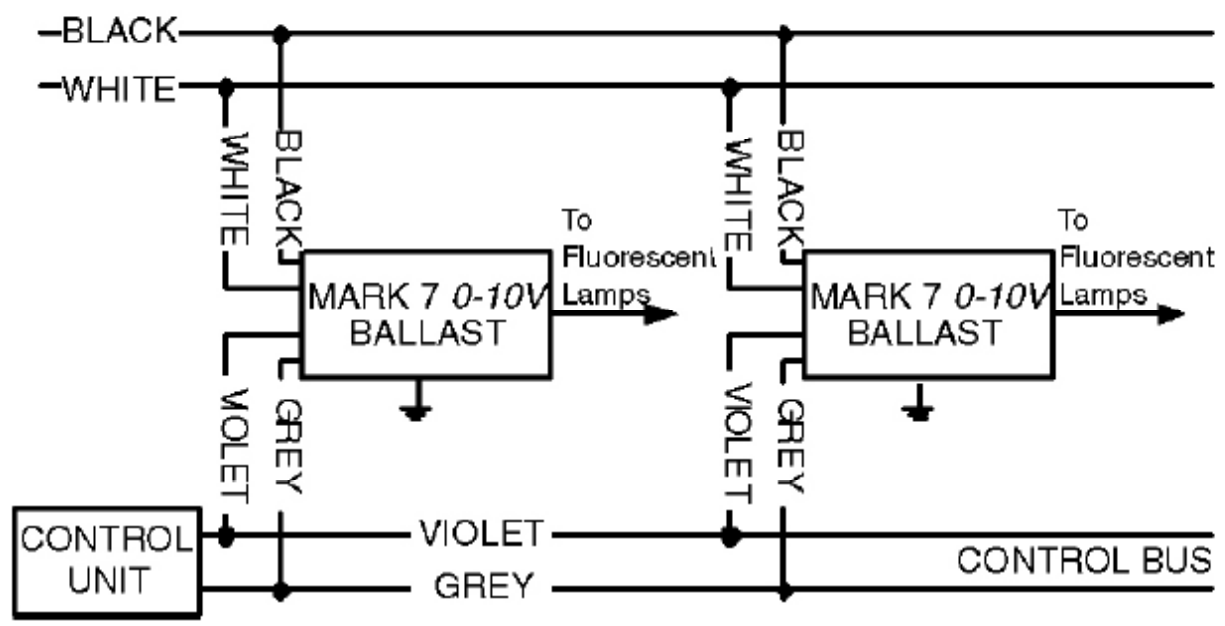

Figure 14: Wiring diagram showing how $0-10$ VDC analog ballasts are wired to a low voltage control loop formed by the violet and grey wires.

\section{Powerline controlled ballasts}

Powerline controlled ballasts do not have any additional wires and they wire to the hot and neutral leads just like normal (non-dimming) ballasts. A dimmer must be installed in the wallbox controlling the lighting circuit (Figure 15). The number of ballasts that can be dimmed on the same dimming circuit depends on the wattage rating of the dimmer. Powerline controlled ballasts are usually used for controlling the lights in individual rooms rather than for entire lighting 
systems. Because they do not require additional wiring, they are often used in retrofit applications.

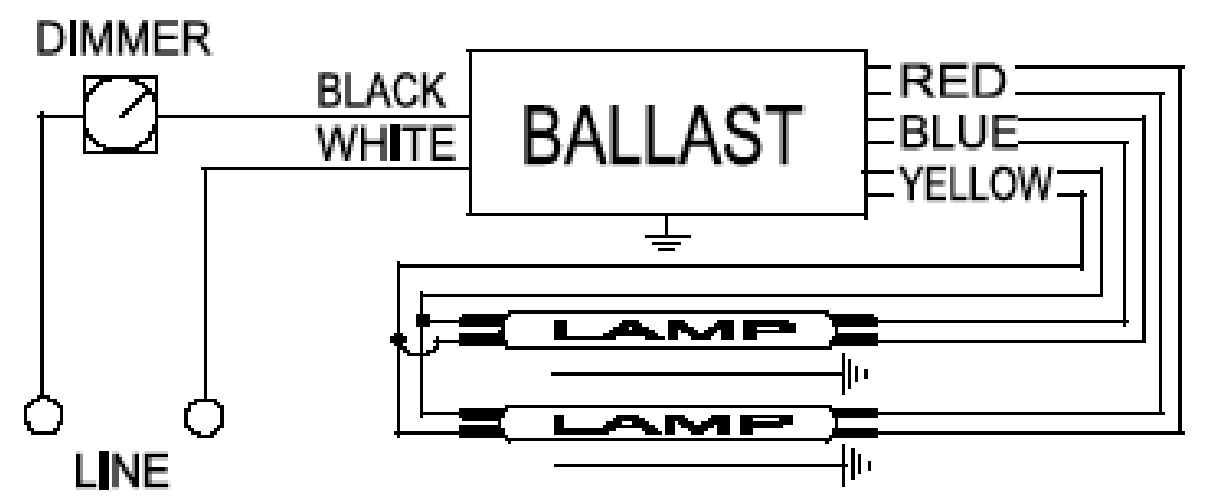

Figure 15: Wiring diagram showing how powerline controlled ballasts are wired to a wall dimmer.

\section{3-Wire controllable ballasts}

3-Wire controllable ballasts have an extra hot lead on them for controlling the ballast dim level. Only one company makes this type of system, which is proprietary.

\section{Digital dimming ballasts}

Although digital ballasts are not a large market at this time (less than $10 \%$ of the already small dimming ballast market), most of today's digital ballasts in the U.S. use the Digital Addressable Lighting Interface (DALI) protocol. DALI is one of several European digital protocols that have been imported into the US over the last few years. All major US ballast companies now sell digitally controllable ballasts that obey the DALI protocol. One major manufacturer (Universal) also sells a competing digital protocol along with their DALI products. 


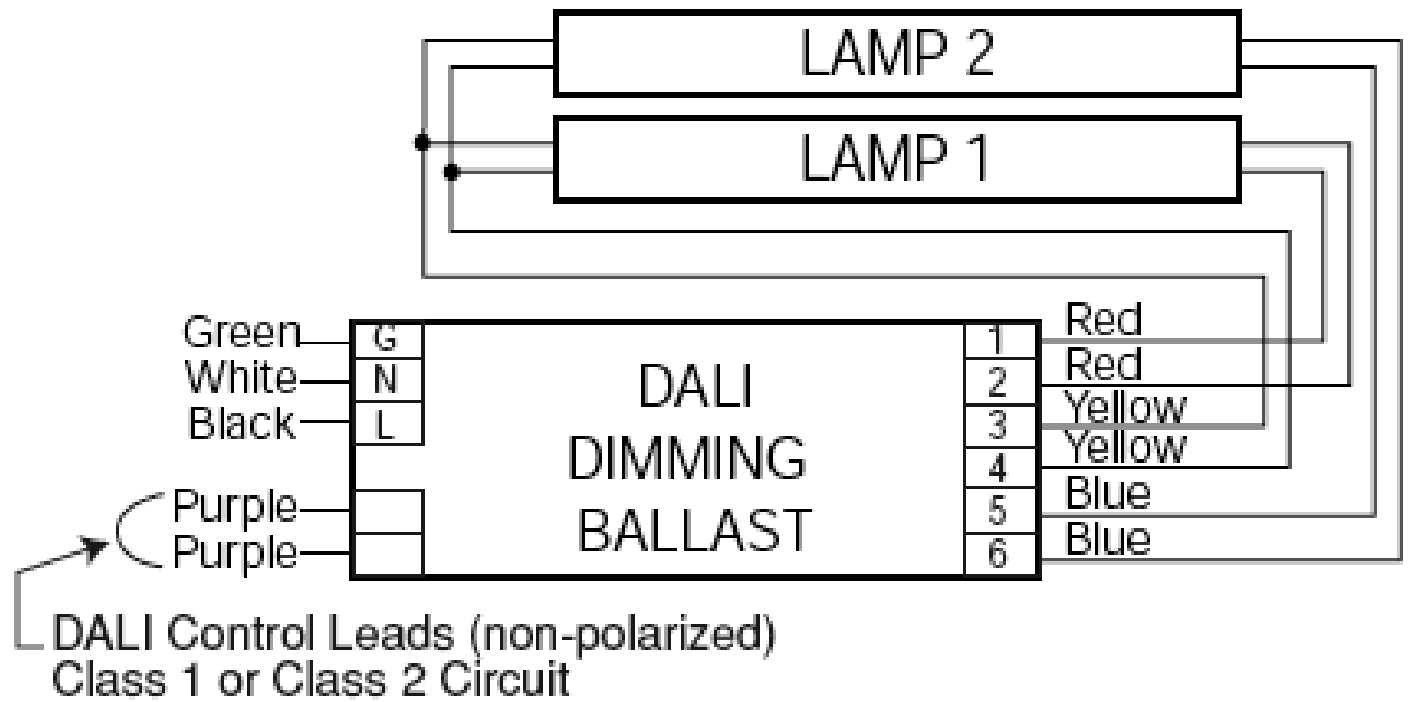

Figure 16: Wiring diagram for a typical DALI ballast. Multiple DALI ballasts are wired in parallel to the DALI control leads. Unlike 0-10 VDC analog dimming ballasts, DALI ballasts are individually addressable.

\subsection{Emerging Technologies to Bridge the Gap}

Although most buildings can potentially participate in DR by manually shedding lighting loads, the advancement in networking technologies and wireless communications make automated and semi-automated DR possible in commercial buildings. In this section, two of Southern California Edison's demand responsive lighting controls projects under their Emerging Technologies Program and Pacific Gas and Electric's Automated Critical Peak Pricing project with LBNL are summarized. All three projects are using different technologies to achieve centrally dispatched demand reductions for the lighting systems. The first two projects concentrate on the use of dimmable ballasts with a centralized lighting control system. The criteria for evaluating both projects are:

A. Central control by a utility;

B. Application of the technologies to 120 Volt and 277 Volt lighting loads;

C. Response time; and

D. Shed amount.

The third project concentrates on the communication infrastructure to reach out to the commercial customers' lighting control system. The existence of a lighting control system in its simplest form is the only prerequisite to participate in the pilot study. Target Corporation's Hayward store shows the possibilities in retail with a well-planned lighting infrastructure. Similar infrastructures also have been used in a semi-automated fashion by other retail chains for DR such as Home Depot [ix]

\subsubsection{LA County Internal Services Division}

The objective of this study was to evaluate demand response effectiveness of the GE Wireless Energy Management technology by controlling lighting and a chiller at a pilot test facility. The 
GE Wireless Energy Management system was installed in LA County Internal Services Division site and controlled by Power Web technologies over the internet $\left.{ }^{\mathrm{X}}\right]$.

The components of the system are:

- Input: Local or Remote User

- User interface: Computer/Web-based software

- Control and Communication: Cellular phone infrastructure, $418 \mathrm{MHz}$ to Lighting Router

- Output: 0-10 volt dimmable ballast

Figure 17 shows the implementation of the system over the existing pathways concept. The demand reduction is initiated at the computer either on site or off site via internet software The signal is sent to a wireless tower using existing cellular phone infrastructure. The lighting router receives the signal from the wireless tower and sends wirelessly over $418 \mathrm{MHz}$ to ballasts controllers. As a part of the project, dimmable ballasts were installed. Each ballast controller controls up to fifty $0-10 \mathrm{~V}$ dimmable ballasts. Their tests show that the response time of this system is between $2-12$ seconds.

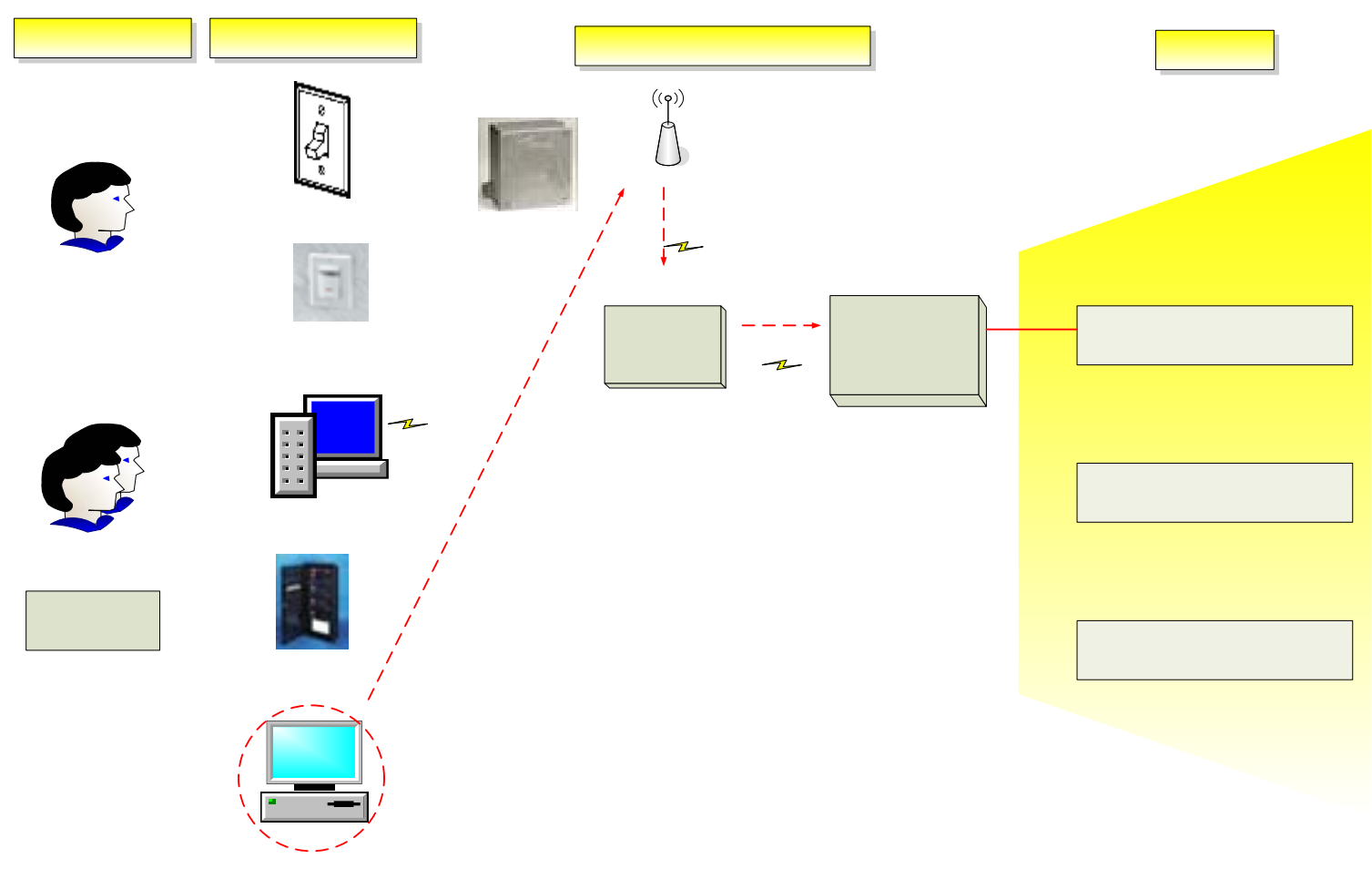

Figure 17: Description of the GE Wireless Energy Management System

The project is a good example of absolute semi-automated demand reduction. To be a fully automated DR, scalability issues have to be examined. It uses the existing wireless infrastructure until the signal is inside the building and transmits the signals to the controller through a less used frequency. 0-10 V wiring is required for the last portion of the installation.

The GE Wireless Energy Management software is administered by Power Web Technologies and access is set up for both the utility and the facility manager to monitor and change the settings of the control system through a web page. In addition, feedback data from the chiller and the lighting panel were made available through the GE Wireless Meter Data Processor. 


\subsubsection{Ganahl Lumber Company}

Universal Lighting Technologies' Demand Control Lighting (DCL) system was installed in a portion of the lighting, mostly in public spaces, of Ganahl Lumber Company, which is a retail hardware store. The test included lighting fixtures on 120 Volt and 277 Volt circuits controlling 72 lighting fixtures on the first and second floors [xi]. The system has the following components displayed in Figure 18:

1. Universal internet software

2. Universal DCL circuit control modules

3. Universal line controlled dimming ballasts

4. Wireless connection to the internet

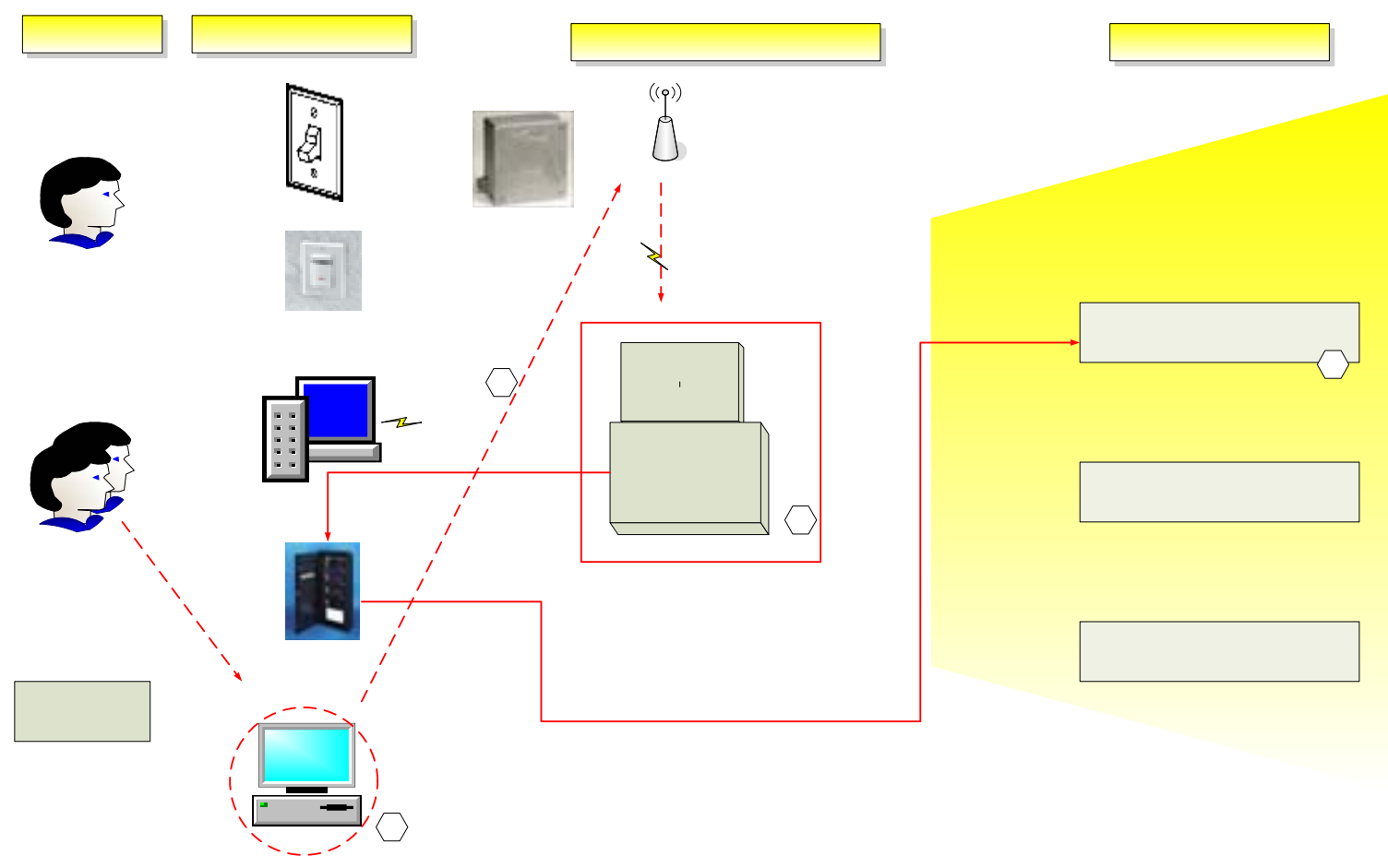

Figure 18: Pathways concept as applied to Ganahl Lumber Company tests

A user issues a command using the Internet Software. A wireless tower then transmits a signal to the master module at the site. The master module receiving the coded message sends a signal to the other control modules to which it is connected. The lighting control modules are located in enclosures adjacent to lighting breaker panels. The controller can send the control message to all ballasts on the circuits to which it is wired. Lighting levels can be set from $100 \%$ to $50 \%$ in $1 \%$ power increments. The ballasts receiving the signal adjust their dimming level according to the instructions issued by the controller. Six ballast controllers were installed for this project. Three controllers were on 277 Volt lighting circuits, and three were on 120 Volt lighting circuits. The response times ranged from 8 to 146 seconds. This is another example of semi-automatic controls using dimmable ballasts.

The best way to compare the demand reduction effectiveness of the above two projects is the demand intensity savings $\left(\mathrm{W} / \mathrm{ft}^{2}\right)$ but neither study mentions per provides information to colstat 
this metric. In the case of the LA County Internal Services, demand savings is stated to be $22.7 \mathrm{~W}$ per fixture when the lights are dimmed from $70 \%$ to $30 \%$. In the case of Ganahl Lumber Company demand savings per fixture when the fixture is dimmed from $100 \%$ to $50 \%$ was $30 \mathrm{~W}$.

\subsubsection{Target Corporation, Hayward}

Target developed a simple strategy based on its infrastructure [xii]. The implementation is mapped onto the pathways concept in Figure 19. Every fourth fixture is circuited together in their Hayward facility. The engineering group in Minnesota is able to issue commands over their network to turn off any of the four circuits in their sales area. For the Critical Peak Pricing (CPP) event days, they chose to turn of one circuit between $3 \mathrm{pm}$ and $6 \mathrm{pm}$.

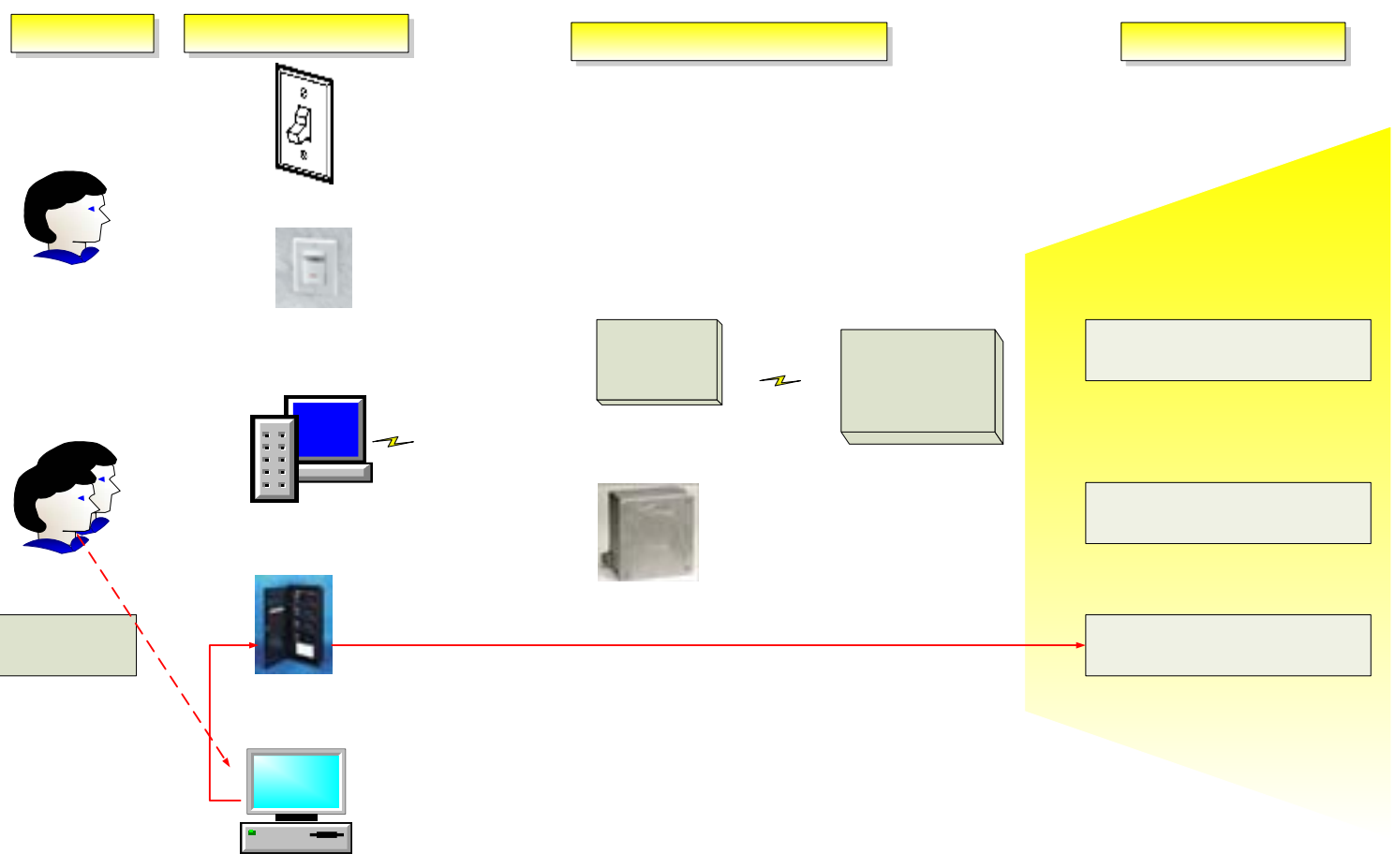

Figure 19: Pathways concept as applied to Target Company Automated Critical Peak Pricing Tests

Due to good initial planning and grouping of the fixtures, although one of every four fixture was switched of, the lighting remained uniform in the sales area. The demand savings result of switching off every fourth fixture was $50 \mathrm{~kW}$ or $0.45 \mathrm{~W} / \mathrm{ft}^{2}$ for the $110,000 \mathrm{ft}^{2}$ store.

There were no direct customer feedback for this site but a store manager was contacted after every event. From the conversations, it was clear that switching every fourth fixture was noticeable and not welcomed, especially closer to the holidays when higher occupancy and sales are expected. 


\subsection{Findings}

\subsection{Context}

\subsubsection{Lighting has a major impact on electricity demand and consumption}

Lighting systems in California commercial buildings consume $30 \mathrm{BkWh}$ annually. Large offices, retail, hospital, miscellaneous and food stores make up nearly $60 \%$ of the total 6 billion square feet of commercial floorspace and use about $75 \%$ of the total lighting energy. Commercial lighting demand is largely coincident with total Statewide peak demand and on peak days is responsible for $\sim 6 \mathrm{GW}$ (30\% of the total demand) during the 2:00 PM- 5:00 PM summer peak (compared to $32 \%$ for air conditioning). Lighting has a major impact on electricity demand in commercial buildings, roughly equal to that of HVAC.

\subsubsection{Lighting wastes energy and unnecessarily strains the electrical grid because lighting controls, especially dimming, are not widely used.}

By drawing less power from the grid when electricity costs are highest, dimming controls enable building lighting loads to become more elastic. But dimming equipment, especially the dimming ballast, costs more than non-dimming lighting and is expensive to retrofit into existing buildings because of the cost of adding control wiring.

\subsubsection{Title 24 has had a positive effect on the lighting wiring in CA buildings.}

Because of Title 24's influence, lighting systems in California buildings have some unique characteristics, especially in how they are electrically wired for bi-level lighting. There are major opportunities to tap the huge reservoir of potential energy and demand savings inherent in the State's wiring infrastructure. EPAct 2005 can help CA ratepayers pay for the installation of new energy-saving controls.

\subsubsection{Internet and wireless are major technology drivers}

The pervasiveness of the Internet and wireless technologies in buildings has led to new opportunities for lighting manufacturers to develop and deploy intelligent lighting controls that realize significant energy savings and reliable demand reduction.

\subsection{Barriers}

\subsubsection{Estimating the energy savings from lighting controls is harder than evaluating the savings from more efficient equipment}

More efficient equipment, such as electronic ballasts, saves energy simply because it is installed. Lighting controls only save energy if they positively impact operational performance. Most utilities have little reliable data on how much energy and demand savings will result from lighting control systems utilizing all lighting control strategies in multiple building types. Without this information, utilities cannot properly calculate how much to rebate lighting control systems in their efficiency programs. Determining the controls savings requires measuring the energy usage both before (baseline) and after installation of controls.

\subsubsection{Adding control wiring to existing buildings is expensive}

It is usually cost-prohibitive to install conventional lighting control components into existing buildings because of the high cost of adding the control wiring. Powerline carrier techniques can 
avoid added wiring but no reliable PLC system has emerged. Wireless dimming ballasts will not be cost-effective for about 2 years.

\subsubsection{Delivering robust systems is harder than manufacturing components}

The US lighting controls industry is fragmented with many component manufacturers but very few manufacturers making entire systems. System integrators find it difficult to assemble and specify robust lighting control systems using components from different vendors.

\subsubsection{Need for proper commissioning not understood.}

Commissioning is the most challenging barrier to the successful deployment of advanced control systems. (Commissioning is the process for ensuring that a control system performs in the building according to design intent). By migrating to intelligent digital controls, the industry can solve the commissioning problem. But the industry currently lacks capability in the critical areas of wireless networking, communications protocols and serious software development.

\subsubsection{Dimming systems are more complicated than non-dimming}

Because dimming systems must work reliably over a wide range of light levels, they are more sensitive to poor lamp holder design, miswiring and sloppy installation. "Standby" losses can reduce the energy efficiency of a lighting control system and the utilities are largely unaware of the issue.

\subsection{Opportunities}

\subsubsection{Advanced lighting controls can be a compelling value proposition}

Dimming will always be more expensive than non-dimming lighting. So to build a convincing economic case for using dimming controls, manufacturers must design robust systems that can implement multiple lighting control strategies. Besides from demand response, the major lighting control strategies are: daylighting, personal controls and occupancy-based controls. Lighting controls should monitor and archive energy data as well as control lighting.

\subsubsection{Personal controls sell}

Dimming according to personal preference is a major driver for lighting controls. Research is increasingly showing the importance of personal lighting controls for occupant comfort, satisfaction and productivity.

\subsubsection{Lighting industry developing controllers}

Manufacturers are starting to produce electronic equipment -- lighting-application specific controllers (LAS controllers) -- that are wirelessly accessible and can control dimmable or multilevel lighting systems obeying different industry-accepted protocols: 1) low-voltage digital, 2) powerline, 3) powerline-carrier or 4) pure wireless communication protocols. LAS controllers are particularly well-suited to retrofitting where it is not cost-effective to add wiring to communicate with downstream lights.

\subsubsection{The major ballast manufacturers have improved the top-end efficiency of their dimming ballasts over the last three years}

These more efficient dimming ballasts are only 10\% less efficient than the most efficient nondimming fluorescent ballasts available today. This demonstrates that the industry has the capability to produce highly efficient dimming ballasts. 


\subsubsection{A concerted program to deploy energy saving lighting controls would be of enormous benefit to California}

A concerted 20 year program to improve the demand responsiveness and energy efficiency of commercial building lighting systems would improve the elasticity of the grid, delay the need to add generation capacity, save Californians billion of dollars in avoided energy charges and significantly reduce greenhouse gas emissions. If advanced lighting controls were to achieve $60 \%$ penetration in the CA commercial building market in 20 years, the State would consume 100 BkWh less electrical energy at a savings to ratepayers of $\$ 12$ billion. Over 20 years, it would reduce greenhouse emissions by $20 \mathrm{MMTCe}--$ the environmental equivalent of removing over 13 million cars from the roads.

\subsection{Recommendations}

Our goal should be to deliver significant and reliable reductions in electric lighting demand and electric energy use throughout California's commercial building stock. This goal is ambitious but it can be accomplished by a coordinated sequence of research and market conditioning activities aimed at timely deployment of robust, cost-effective, energy efficient, demand responsive lighting systems.

\subsection{Set an ambitious, but practical, goal}

Establish an ambitious but practical goal for significantly reducing lighting energy consumption in California commercial buildings. Our vision is: "Every light should be dimmable, addressable and affordable".

\subsection{Learn from the experience with the electronic ballast}

In the late 1980s and early 1990s, PG\&E used the "Penrose List" to qualify electronic ballasts for their active rebate programs. By setting performance objectives appropriate to their Energy Efficiency Program objectives, the Penrose List helped PG\&E to transform the California market from magnetic to electronic ballasts and save the State billions of dollars over the last 15 years. We propose below an equivalent vehicle for transforming the market to energy-saving lighting controls. The Performance Metrics list below is necessarily more complex than Penrose's, which only had to characterize a simple electronic ballast.

\subsection{Develop a uniform test protocol}

Develop, refine and promulgate a uniform test protocol for determining the suitability of specific emerging lighting control systems for delivering reliable demand response and implementing all major lighting control strategies.

1. Formulate a building wide, robust, versatile, strategic controls framework that provides a "building-entry-to-lighting end-use" control signal pathway to communicate with and control any light fixture in a building.

2. Detail in matrix form the functional requirements for implementing demand responsive and all the major energy efficiency strategies, including daylighting, personal controls, tunable lighting and occupancy detection.

3. Develop a list of appropriate, quantifiable performance metrics that a given control system must satisfy in order to "qualify" it for implementing different combinations of efficiency and demand strategies.

4. Expand the Functional Requirements and associated Performance Metrics lists to cover additional technical issues relevant to Emerging Technology Programs such as dimming ballast characteristics, susceptibility to outside interference, and scaling controls from the room level to building-wide control. 
5. Develop procedure for testing how well candidate lighting controls systems satisfy the Performance Metrics list.

\subsection{Develop data base of measured energy and demand savings}

Utilities need a consistent, reliable methodology for evaluating the energy savings and demand shed potential for various combinations of lighting control strategies in different building applications, regardless of networking technology. Develop a database of the energy and demand savings from controls as measured by early adopters in testbeds and pilot installations. With such a database, utilities will be able to appropriately incentivize the installation of energy savings controls in all building types. Make the database accessible to utilities and emerging technology program managers.

\subsection{Make appropriate code revisions}

Encourage the development of cost-effective dimming through carefully crafted code revisions. Some of these revisions include:

1. Replace Ballast Efficacy Factor (BEF) with Relative System Efficacy (RSE) as a sensible figure of merit for comparing lamp/ballast system efficacy across all major lighting types. RSE should be used for the 2008 Revision of Title 24 .

2. Set appropriately challenging RSE targets to include in subsequent revisions to Title 24 and 20 codes to encourage the adoption of efficient dimming technologies. Appendix $\mathrm{C}$ provides an example of how RSE target should be set for dimming ballasts for the 2008 revisions. This recommendation was sent to CEC Staff October, 2006.

3. Consider requiring the installation of generic control cabling for all new buildings. This should be enacted in the 2008 Revision to Title 24.

4. Consider requiring dimming ballasts for all applications for the 2011 Revision to Title 24.

\subsection{Leverage EPAct 2005}

Promulgate advice as to how California businesses can best take advantage of the energy efficient lighting installation tax incentives offered as part of National EPA 2005 legislation. Provide advice to end-users who want to use the tax incentives from EPAct 2005 to upgrade lighting systems. Up to $\$ 0.60 / \mathrm{sf}$ are available for lighting efficiency measures that are $50 \%$ of ASHRAE 90-1. A good summary of this topic is available at: http://www.aboutlightingcontrols.org/education/papers/taxdeduction3.shtml. 


\subsection{References}

[i] D\&R International Ltd, "2005 Buildings Energy Data Book”, Prepared for the Energy Efficiency and Renewable Energy, U.S. Department of Energy, August 2005

[ii] Rubinstein, F. (1991). "Automatic Lighting Controls Demonstration: Long Term Results", Final Report for Pacific Gas \& Electric, Lawrence Berkeley National Laboratory.

[iii] Piette, M.A., D.S. Watson, N. Motegi, and N. Bourassa, Findings from the 2004 Fully Automated Demand Response Tests in Large Facilities, LBNL-58178. September 2005

[iv] Commercial Buildings Energy Consumption Survey 2003. Available online at: http://www.eia.doe.gov/emeu/cbecs/

[v] Internal CEC Lighting Technology spreadsheet based on utility Commercial End Use Surveys, California Energy Commission, 2002

[vi] Rubinstein, F, The Usefulness of Bi-Level Switching, Lawrence Berkeley National Laboratory Technical Note, LBNL-44281, Revised August 1999. http://btech.lbl.gov/papers/44281.pdf

[vii] ADM Associates, Lighting Controls Effectiveness Assessment: Final report on Bi-Level Lighting Study, May 2002.

[viii] DiLouie C, The Next Generation of Electronic Lighting Systems: Smaller, Smarter and Greater Energy Savings, 2003.

http://www.aboutlightingcontrols.org/education/papers/ballasts.shtml

[ix] Chris Smith, New York State Energy Research and Development Authority Gary Epstein and Mark D'Antonio, Energy \& Resource Solutions, Inc. Demand Response Enabling Technologies and Case Studies from the NYSERDA Peak Load Reduction Program, ACEEE Summer Study 2004.

[x] Edison Design and Engineering Services, Assessing the Demand Response Capabilities of a Remotely Controlled, Continuous Dimming Lighting System, March 31, 2006

[xi] Edison Design and Engineering Services, Assessing the Demand Response Capability of a Remotely Controlled, Stepped Dimming Lighting System, March 15, 2006

[xii] Kiliccote S., Piette M.A. and Hansen D., Advanced Controls and Communications for Demand Response and Energy Efficiency in Commercial Buildings, Proceedings of Second Carnegie Mellon Conference in Electric Power Systems, Pittsburgh, PA. LBNL Report 59337. January 2006 
Appendix A

State-of-the-Art Review of the Performance Characteristics of Dimming Ballasts 


\section{Introduction}

We show that the "full output" efficiency of the newer dimming ballasts is beginning to approach the efficacy of non-dimming program start ballasts but is still about $10 \%$ lower than "high performance" Instant Start ballasts. We present the concept of "standby losses" in dimming ballasts and show that most manufacturers do not identify the standby losses in their product literature. Then we present data which compares the relative system efficiency of all nondimming and dimming ballasts all on one graph so that the efficiencies of different models can be easily compared.

\section{Dimming Ballast Performance Issues}

Dimming ballasts share most of the same performance characteristics as their non-dimming brethren, but the dimming capability means that dimming ballasts have some additional properties that specifiers must consider if they are to use dimming ballasts effectively in the design process.

If specifiers are to choose between different dimming ballast types based on efficiency, they need specific data from dimming ballast manufacturers. Many dimming ballast manufacturers already provide ballast factors and input power in their technical data sheets. To determine the relative light output and actually input wattage drawn at full light output requires that the manufacturer list 1) the ballast factor and 2) the actual system input wattage. To determine the relative light output and drawn power under maximally dimmed condition, requires that manufacturers report 1) ballast factor at minimum light output and 2) actual power drawn at minimum light output. (Figure 1 shows an excerpt from a typical ballast manufacturer's catalog that lists all the relevant data). Unfortunately, some manufacturers do not supply this data, which reduces the specifier's ability to predict the energy performance of a system. Because a modern dimming system may spend considerable time at this minimum output level, manufacturers should provide the data that identifies exactly what this usage is.

\begin{tabular}{|c|c|c|c|c|c|c|c|c|c|c|c|c|}
\hline \multicolumn{2}{|c|}{ Lamp Data } & \multirow{2}{*}{$\begin{array}{l}\text { Min. } \\
\text { Starting } \\
\text { Temp. } \\
\left({ }^{\circ} \mathrm{F} /{ }^{\circ} \mathrm{C}\right)\end{array}$} & \multirow{2}{*}{$\begin{array}{l}\text { Input } \\
\text { Volts }\end{array}$} & \multirow{2}{*}{$\begin{array}{l}\text { Catalog } \\
\text { Number }\end{array}$} & \multicolumn{2}{|c|}{ Certifieations } & \multirow{2}{*}{$\begin{array}{l}\text { Line } \\
\text { Curren1 } \\
\text { (Amps) }\end{array}$} & \multirow{2}{*}{$\begin{array}{c}\text { Input } \\
\text { Power } \\
\text { ANSI } \\
\text { (Watts) } \\
\text { maximin }\end{array}$} & \multirow{2}{*}{$\begin{array}{c}\text { Ballast } \\
\text { Factor } \\
\text { maximin }\end{array}$} & \multirow{2}{*}{$\begin{array}{l}\text { Max. } \\
\text { HD \% } \\
\text { at full } \\
\text { llght } \\
\text { ulpout) }\end{array}$} & \multirow{2}{*}{$\begin{array}{l}\text { Mln. } \\
\text { Power } \\
\text { Factor }\end{array}$} & \multirow{2}{*}{$\begin{array}{c}\text { Dim./ } \\
\text { Wiring } \\
\text { Diagram }\end{array}$} \\
\hline Number & Watts & & & & (4) & (15) & & & & & & \\
\hline \multicolumn{13}{|c|}{ F32T8, FB031T8, F32T8/U6 } \\
\hline 1 & 32 & $50 / 10$ & $\begin{array}{l}120 \\
277\end{array}$ & ZT-132-SC & $\frac{\gamma}{r}$ & $\frac{\gamma}{r}$ & $\begin{array}{l}0.30 \\
0.13\end{array}$ & $35 / 8$ & $\begin{array}{l}1.00 / \\
0.03\end{array}$ & 10 & 0.99 & Fig. B/55A \\
\hline 2 & 32 & $50 / 10$ & $\begin{array}{l}120 \\
277\end{array}$ & ZT-2S32-SC & $\frac{\gamma}{r}$ & $\frac{\gamma}{r}$ & $\begin{array}{l}0.57 \\
0.24\end{array}$ & $70 / 14$ & $\begin{array}{l}1.00 / \\
0.03\end{array}$ & 10 & 0.99 & $\mathrm{Fig} \cdot \mathrm{B} / 56 \mathrm{~A}$ \\
\hline 3 & 32 & $50 / 10$ & $\begin{array}{l}120 \\
277\end{array}$ & ZT-3S32-SC & $\frac{\gamma}{r}$ & $\frac{\gamma}{r}$ & $\begin{array}{l}0.86 \\
0.37\end{array}$ & $102 / 20$ & $\begin{array}{l}1.00 / \\
0.03\end{array}$ & 10 & 0.99 & $\mathrm{Fig} . \mathrm{B} / 57 \mathrm{~A}$ \\
\hline 4 & 32 & $50 / 10$ & $\begin{array}{l}120 \\
277\end{array}$ & ZT-4S32 & $\frac{\gamma}{r}$ & $\frac{\gamma}{r}$ & $\begin{array}{l}0.98 \\
0.42\end{array}$ & $116 / 25$ & $\begin{array}{l}0.88 / \\
0.03\end{array}$ & 10 & 0.99 & Fig. D/16A \\
\hline
\end{tabular}

Figure 1: The orange box highlights the input power and ballast factor data from a ballast manufacturer's catalog.

\section{Standby Losses}

Some dimming ballasts also have the ability to switch off the connected lamps without switching off the dimming control circuitry. The lamps are usually switched off by signaling the ballast over 
a digital bus (e.g., DALI) or, rarely, a 0-10 VDC control loop. When the ballast is in this "standby" state, the lamps produce no light but the lamp/ballast/control system still consumes a small amount of power. This "standby" loss is analogous to the standby losses in modern appliances. For a typical 2 lamp T8 ballast drawing a nominal 60 watts at full output, the standby loss is between 1-6 watts. We have found no ballast manufacturer that quotes the standby loss value in their product literature.

\section{Dynamic Dimming Range}

The information provided by most dimming ballast manufacturers is adequate to determine the energy performance of the ballast under full power and fully dimmed modes. Furthermore, most dimming ballasts operate linearly between these two extremes. Practically however, the control system may impose additional limitations on the actual dimming range achievable in the field.

To illustrate these concepts more clearly, we use a particular dimming ballast (Sylvania's new PowerSense dimming ballast) as an example. As stated above almost all dimming ballast manufacturers list the full light output ballast factor and the associated power draw at full output. Many, including Sylvania also list the minimum values: i.e., the ballast factor at full dim and the associated power draw at that lowest level. These data for the PowerSense ballast is plotted in Figure 2.

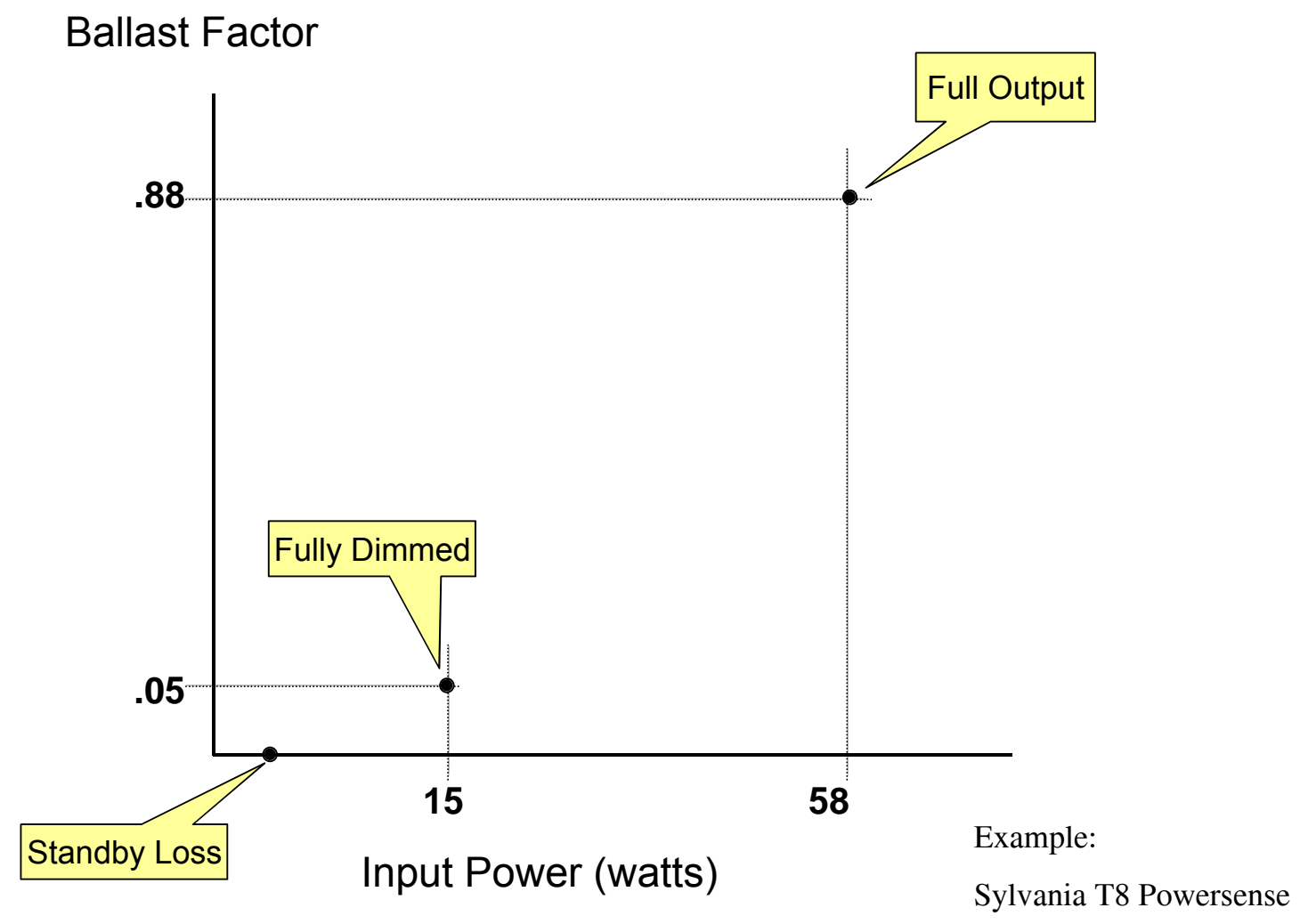

Figure 2: Key ballast performance data plotted with input power on horizontal axis and ballast factor on the vertical axis. The data shown is for the Sylvania PowerSense ballast. 
What is the performance of the ballast between the two extreme? Most dimming ballasts that we have measured have linear performance between these two extremes. For example, below is the relationship between ballast factor and input power for three different DALI dimming ballasts that we measured in our laboratory. Note that the relationship between light output and input power is quite linear. The "power-light" lines for each ballast are largely overlapping with the main difference being the bottom level provided.

\section{Relative I nput Power vs. Relative Light Output for 3 DALI Ballasts}

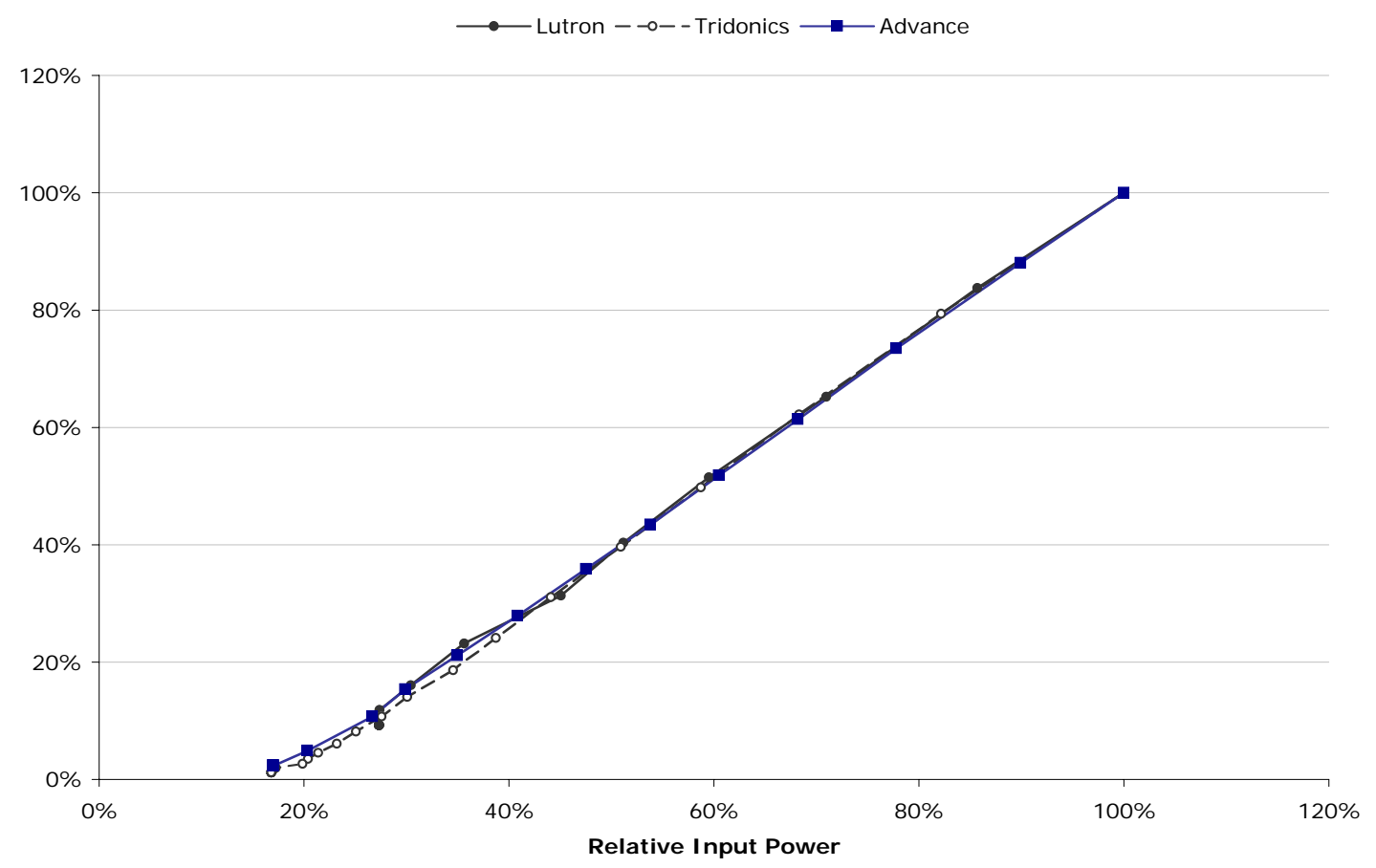

Figure 3: Linear relationship between relative input power and relative light output for three different DALI ballasts from different manufacturers.

From Figure 3, we see that a line drawn between the two extreme points is a very good approximation of the actual dimming curve. This curve is best measured in the lab where temperature conditions around the lamps can be well controlled. The dimming curve can be measured in the field but it is more difficult.

Fitting a line to the two extreme points for our current example is shown in Figure 3. As stated earlier the relationship between light output and input power is linear between the two points, but not proportional. A proportional relationship (as shown by the line labeled "ideal dimming line of constant efficacy" in Figure 3) would be preferable since that would mean that the lamp-ballast system efficacy (expressed in lumens per watt) does not drop as the ballast dims the lamps. Unfortunately, this will not be true of any practical dimming ballast for fluorescent lamps because of the need to maintain lamp filament power as the lamps dim. 


\section{Ballast Factor}

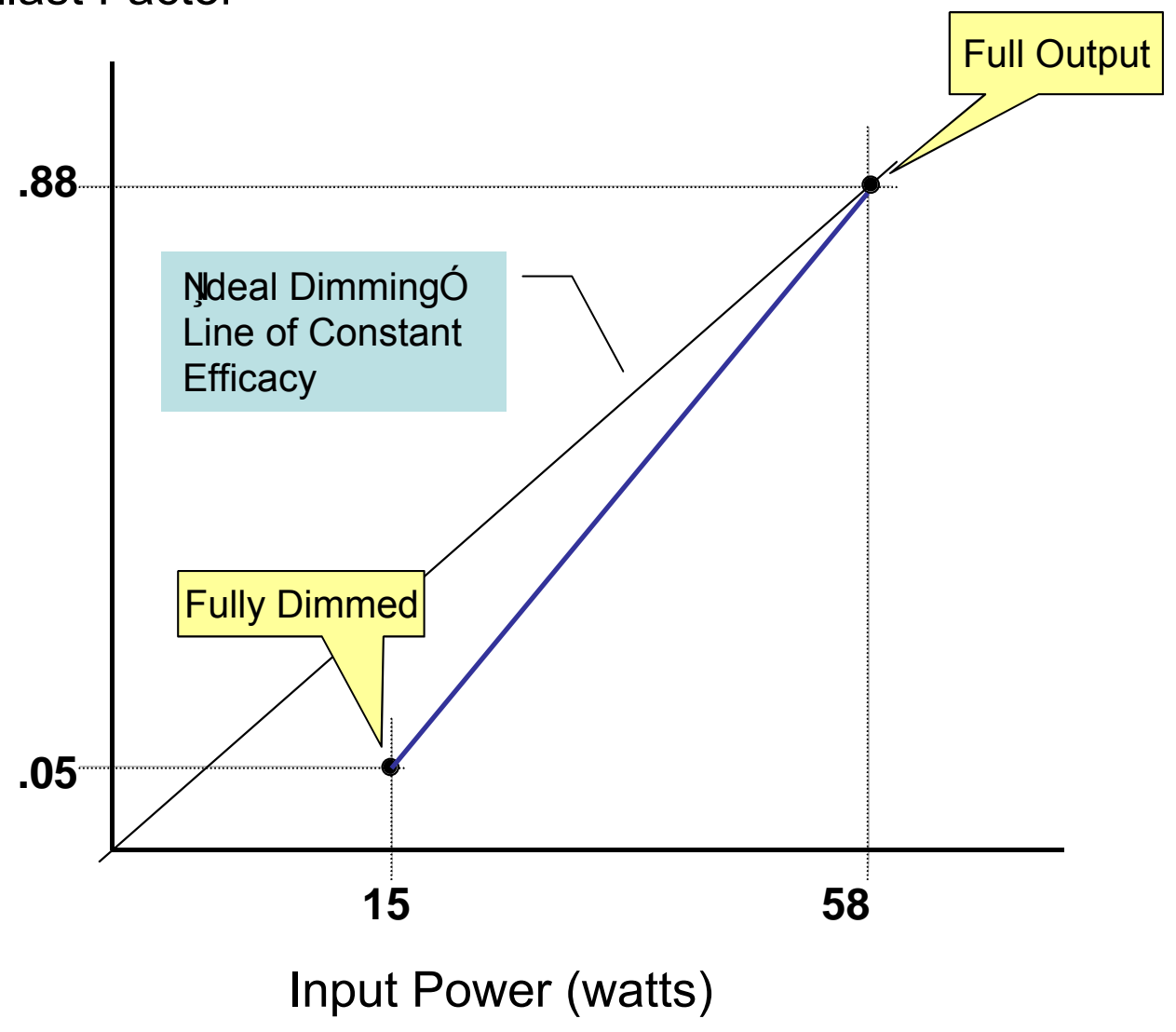

Figure 4: Most dimming ballasts have a linear relationship between input power and ballast factor. Ideally, the relationship between input power and ballast would be proportional as indicated by the line marked "line of constant efficacy."

It is important to realize that the "top" and "bottom" points are a property of the ballast, not the control system. The control system may impose additional limitations that further restrict the actual achievable dimming range.

\section{Actual Dimming Range}

In operation, it is often desirable to restrict the dimming range either by limiting high bright the lamps can go or limiting how low they can be dimmed. As an example, consider the PowerSense ballast. In order to prevent unstable operation at full dim, the installer may set the full dim level to $10 \%$, not $5 \%$. Also, if a space is overlit, the dimmer may be trimmed on the top end so that it can only go to .80 rather than the full .88. usually, these trim adjustments are made on the control system, not the ballast. Once these commissioning adjustments have been made, the dimming range is more restricted. As shown in Figure 1 for our example, the imposition of a .8 "top end trim" and a .1 "bottom end trim" restricts the power reduction range that can be practically achieved. Without the trims, the system power could be maximally reduced from 58 to 15 watts (43 watts or $74 \%$ relative power reduction). With the trims in place, the system power can only be maximally reduced from 54 to 17 watts ( 37 watts or $68 \%$ relative power reduction). 


\section{Ballast Factor Actual available power reduction range (18 to 52 watts)}

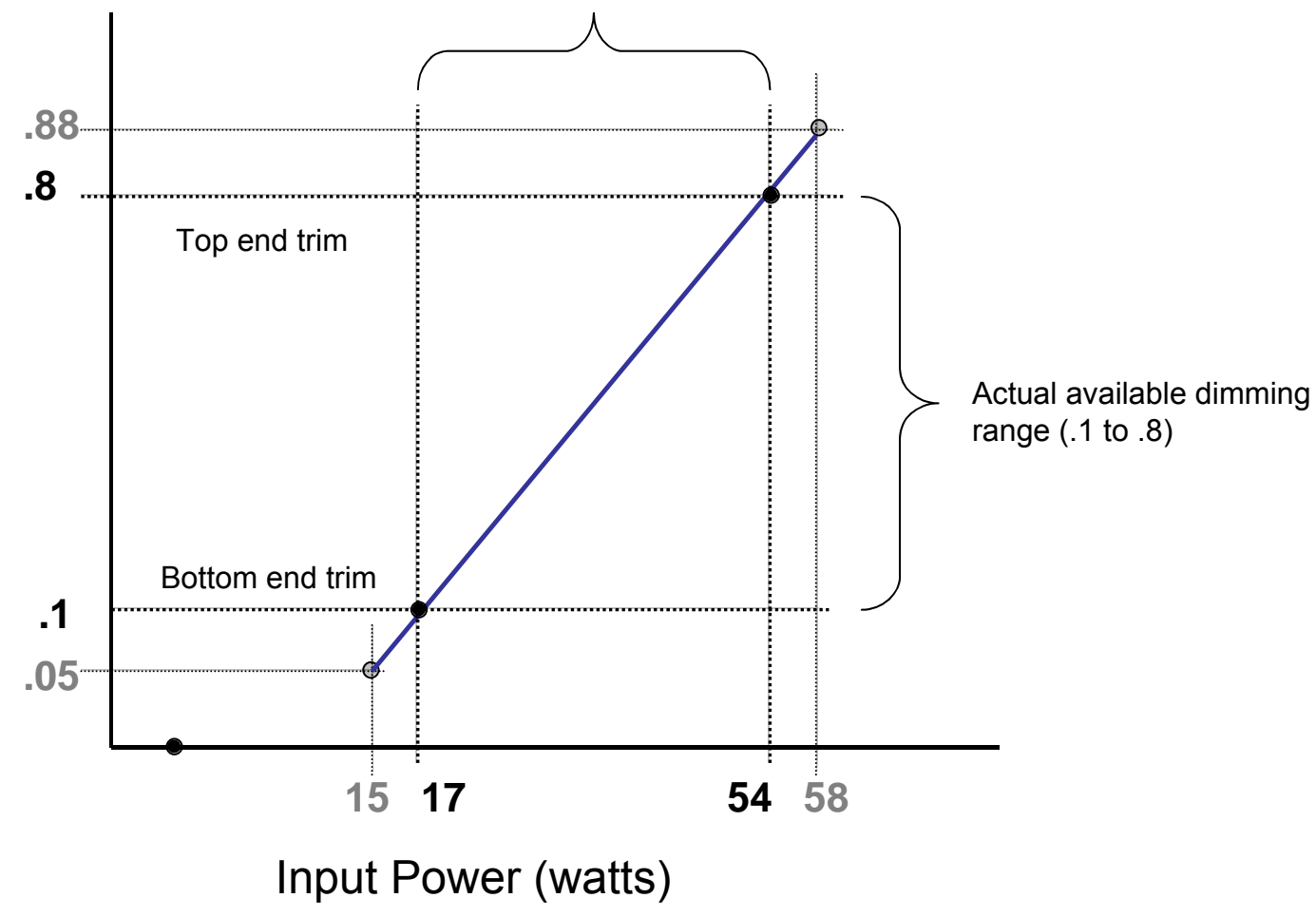

Figure 5: Graph showing how "trimming" top and bottom of the dimming range reduces the effective power reduction achievable by the dimming ballasts.

\section{Relative System Efficiencies}

In this section, we present the results of a technical data search in which we gathered the most recent performance data on commercially available dimming ballasts. The purpose of this exercise was to compare the "full output" system efficacy of modern dimming ballasts and compare them the system efficacies of non-dimming "high performance" Instant Start ballasts and Program Start ballasts. The data for the Instant Start and Program Start ballasts was obtained from the Consortium for Energy Efficiency (ref). Ballast performance data for all commercially available dimming ballasts was collected as part of this project using manufacturer's data as the source.

The justification for using Relative System Efficiency (RSE) for this inter-comparison rather than the more conventional (but flawed) Ballast Efficacy Factor (BEF) is given in an Appendix.

Table 1 compares the relative system efficiencies (RSE) of the current crop of available Instant Start, Program Start and Dimming ballasts on an equal basis. The average RSE of currently available Program Start ballasts is approximately 5.5\% lower than the average RSE for High Performance Instant Start ballasts. 


\begin{tabular}{|c|c|c|c|}
\hline & Instant Start & Program Start & Dimming \\
\hline Number of Ballasts in Sample & 95 & 21 & 30 \\
\hline Mean RSE & 1.02 & 0.964 & 0.917 \\
\hline Standard Deviation & 0.02 & 0.036 & 0.049 \\
\hline Maximum RSE & 1.06 & 1.03 & 1.006 \\
\hline Minimum RSE & 0.986 & 0.91 & 0.82 \\
\hline $\begin{array}{l}\% \text { Reduction in Mean RSE } \\
\text { Relative to Instant Start }\end{array}$ & $0.0 \%$ & $5.5 \%$ & $10.1 \%$ \\
\hline
\end{tabular}

In the figure below we graph the relative system efficiencies of 95 "High Performance" T8 ballasts, 21 Program Start ballasts and 30 Dimming ballasts.

Relative System Efficiencies Compared for I nstant Start, Program Start and Dimming Ballasts

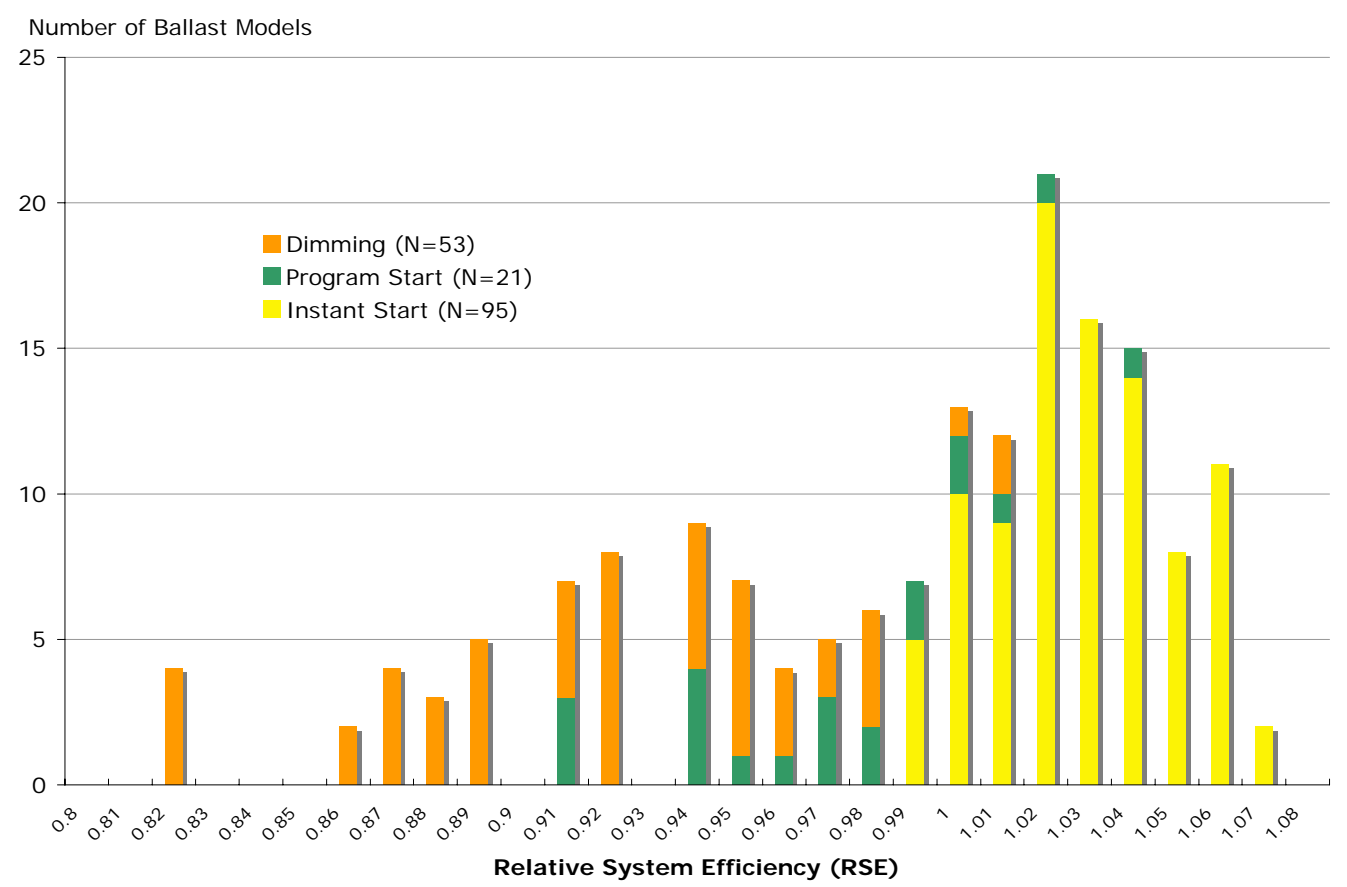

Figure 6: Relative system efficiencies for high performance instant start, program start, and dimming ballasts.

\section{Summary}

Dimming ballasts have improved considerably over the last few years. The "full output" efficiency of modern dimming ballasts is about $10 \%$ higher than older dimming ballast designs. The recent performance data we collected shows that the efficiency of some modern dimming 
ballasts only trails modern Program Start ballasts by a modest 3-5\%. Even the most advanced dimming ballasts are about 10\% less efficient than "High Performance" Instant Start ballasts but this gap has narrowed. Not all dimming ballast manufacturer's have improved their dimming ballast product performance as evidenced by the large number of dimming ballasts with poor efficiency (under 0.9 RSE). It suggested that California impose equipment efficiencies on dimming ballasts via Title 20 that eliminate those ballasts that perform below a specified RSE.

Many, but not all, dimming ballast manufacturers supply the data necessary to evaluate the energy performance of competing dimming ballasts. However, some manufacturers do not list the performance at the bottom of the dimming range. Manufacturers should list this data as the performance of a dimming system cannot be predicted without it. 


\section{Appendix B}

Projected Energy Savings and Market Potential for Wireless Lighting Controls in the State of California 


\section{Introduction}

This Appendix presents an estimate of the energy savings and market potential from installing wireless lighting controls into CA commercial buildings. A concerted program to install wireless lighting controls in $60 \%$ of CA commercial buildings by 2025 would save the State over \$15 billion in avoided energy costs and create a new \$2 billion market for energy-saving lighting controls. The environmental consequence of the program would be the equivalent of removing 14 million cars from California roads.

\section{Background}

In the 1990s, magnetic fluorescent ballasts, which previously dominated US commercial buildings, were supplanted by more efficient electronic ballasts (and T-8 fluorescent lamps). Energy consumers saved of billions of dollars in energy costs as a result of this transformation to efficient electronic ballasts. We hypothesize that this prior success can not only be duplicated but even surpassed by a concerted program to retrofit all California commercial buildings (existing as well as new) with wireless lighting controls over a 20 year time period. This memo explores the implications of such a transformation on California energy consumption and the expected cost savings to the State. The memo also provides a quantitative estimate of the business opportunities for lighting companies that would be created as a result of greatly expanded sales of new lighting technologies to service this ambitious infrastructure modernization program.

\section{California Commercial Lighting Energy Usage}

To determine the total energy used for lighting in CA commercial buildings, we use [MB spreadsheet] for CA lighting energy consumption in 2000. This quotes California's commercial building lighting was $24 \mathrm{BkWh}$ in 2000, which extrapolates to $26 \mathrm{BkWh}$ in 2004 and includes all lighting sources used in commercial buildings. To develop the baseline ("business-as-usual") scenario, we assume that this usage would increase each year in proportion with the expected increase in CA commercial floor space. For projected floor space increase, we have used [xiii] and [xiv] and scaled it to California's commercial floor space.

The time horizon for the current analysis is $2005-2025$. Over this 20 year time period, the CA building stock is projected to increase by $33.6 \%$ or an average annual growth of $1.7 \%$. From the lighting energy consumption for 2000 and the size of the commercial floor space the same year, we can calculate that the average lighting energy consumption per unit area (Energy Use Intensity or EUI) is $5.38 \mathrm{kWh} / \mathrm{ft}^{2}$-yr. To estimate the expected growth in energy consumption from building lighting systems in the absence of lighting controls, we forecast that the lighting EUI would slowly improve due to gradual replacement of incandescent with fluorescent sources as well as evolutionary improvements in fluorescent lighting system efficacy. Between 2005 and 2025, we estimate that the lighting EUI would improve by 5\%.

\section{Assumptions}

The assumptions made in this analysis are given in the following Table 1. A more thorough analysis would estimate uncertainties for all key factors and perform a sensitivity analysis that permutes the calculation across the range of expected uncertainties of the key variables.

\begin{tabular}{|c|c|c|}
\hline Parameter & Estimate & Unit \\
\hline CA Lighting Energy Consumption 2004 & 26 & $\mathrm{BkWh}$ \\
\hline Total CA Commercial Floor space 2004 & 4.98 & Billion square feet \\
\hline Lighting Energy Use Intensity 2004 & 5.38 & $\mathrm{kWh} / \mathrm{ft}^{2}-\mathrm{yr}$ \\
\hline Forecasted improvement in EUI & $0.25 \%$ & Per annum \\
\hline
\end{tabular}




\begin{tabular}{lll}
\hline Cost of Electricity & 0.14 & $\$ / \mathrm{kWh}$ \\
Energy Savings from Wireless Controls & 50 & $\%$ \\
Value of Dimming Ballast & 25 & $\$ /$ ballast \\
Area Covered by One Ballast & 64 & Square Feet \\
Conversion from Primary to Secondary Energy & 91 & BkWh per Quad \\
\hline
\end{tabular}

\section{Market Penetration Scenarios}

We hypothesize that building lighting systems can be largely converted to efficient, intelligent lighting by installing wireless lighting controls in a significant fraction of commercial floor space. We model the expected penetration rate after the national experience with the electronic ballast in the 1990s. Between 1990 and the present, the electronic ballast largely replaced the magnetic ballast in US commercial buildings. As of 2005, electronic ballast sales seem to be saturating at about $60 \%$ of the total ballast market [xv]. The historical growth of the electronic ballast is given in Figure 1.

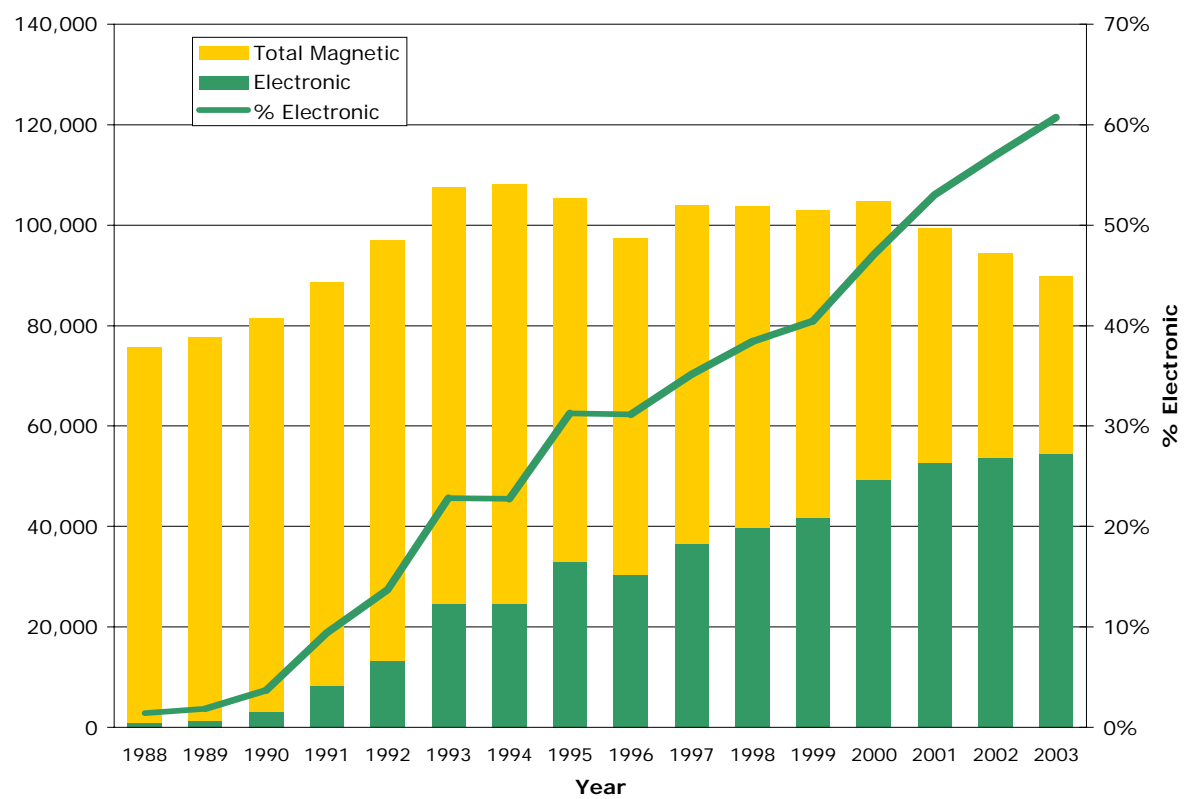

Figure 1: The historical growth of the electronic ballast from 1988 - 2003 based on US Census data [xv]. As of 2003 electronic ballasts were over $60 \%$ of annual US ballast shipments effectively displacing magnetic ballasts in most commercial applications.

At the time of this writing, annual sales of electronic ballasts are about $60 \%$ of the total annual ballast shipment. In terms of in-place US building stock, electronic ballasts and T-8 fluorescent lamps are perhaps $50 \%$ of the fluorescent lighting market. (Presumably, this will approach the yearly saturation (60\%) in a few years). 


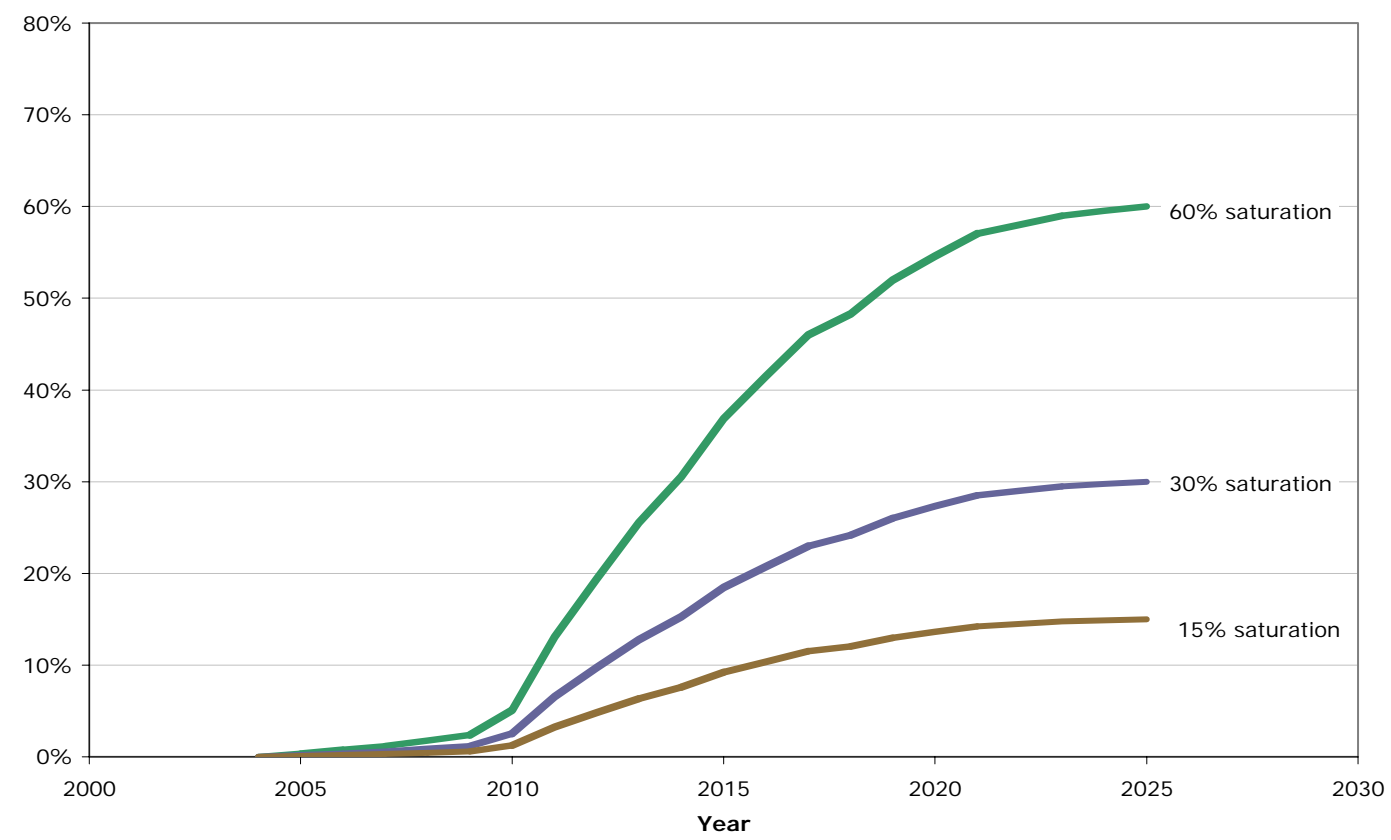

Figure 2: Three market penetration scenarios for wireless lighting controls. The $60 \%$ saturation scenario duplicates the market success of the electronic ballast. The other scenarios are less aggressive.

For the wireless lighting controls technology, we model three penetration scenarios:

High: Saturating at $60 \%$ of standing building stock by 2025

Medium: Saturating at 30\% of standing building stock by 2025

Low: Saturating at $15 \%$ of standing building stock by 2025

The High scenario would be approximately the same aggressive trajectory as the electronic ballast. (We have smoothed the actual electronic ballast yearly sales to remove some year-to-year anomalies). The $30 \%$ and $15 \%$ saturation curves are generated by scaling the $60 \%$ curve proportionally. Note that the shape of the curve consists of three parts: an initial slow rise in the use of controls for the first five years, a rapid increase in deployment for the next $10-12$ years and finally a $3-5$ year leveling off period as the market saturates at its final level. This penetration rate is more realistic in terms of market behavior than assuming the usual s-shape curve.

\section{California Energy Savings Impacts}

Using these penetration curve and the assumptions below, one can calculate the impact of wireless lighting controls on the energy use in the CA commercial building sector. 
I mpact of Wireless Lighting Controls on CA Commercial Lighting

Energy (Three Market Penetration Scenarios)

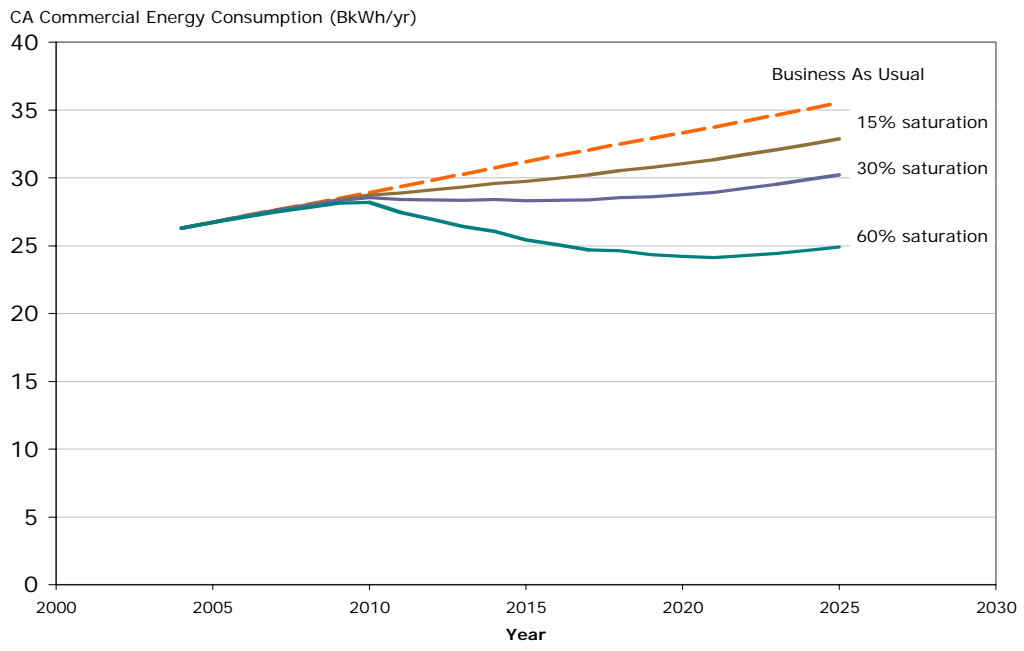

Figure 3: The estimated energy savings from deploying wireless lighting controls in CA buildings according to three different penetration scenarios.

Figure 3 shows the impact of the three scenarios on projected energy consumption for lighting over the next 20 years. In the $30 \%$ penetration scenario, growth is largely checked until 2025, when usage grows again but at a lower rate than before the transformation. If lighting controls can achieve the same $60 \%$ saturation as electronic ballasts, lighting energy consumption nationally would decrease from its current level and remain lower by the end of the transformation.

Now we examine the implications of each scenario, separately, on California energy usage over the next 20 years and simultaneously examine the benefit to the lighting control industry in terms of greatly increased sales of dimming electronic ballasts required to fuel the lighting system conversion.

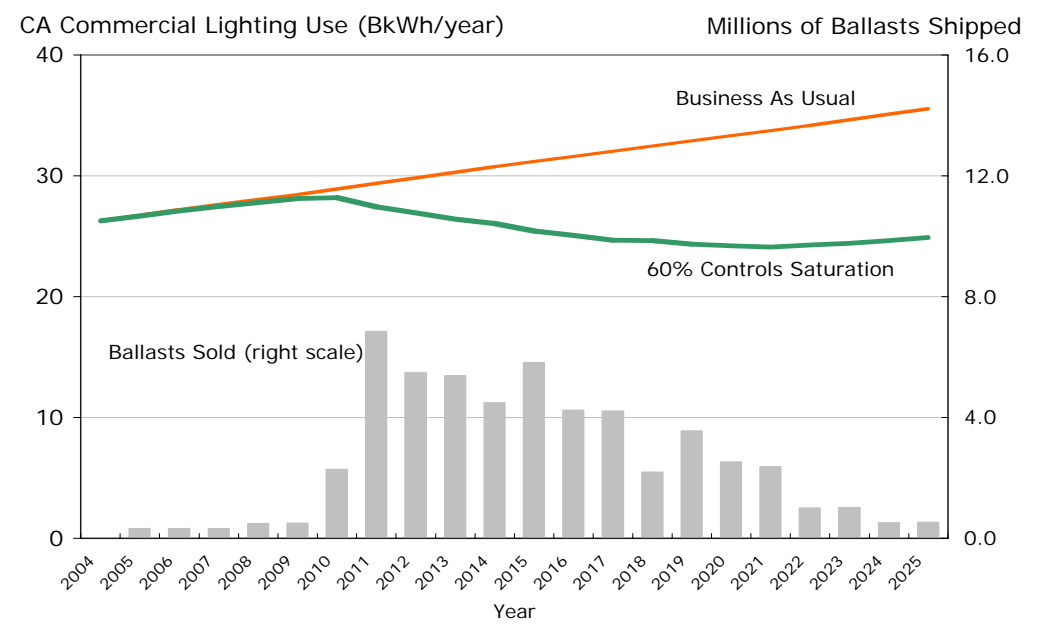

Figure 4: The estimated California energy savings (left hand scale) from deploying wireless lighting controls in $60 \%$ of CA building stock by 2025 . The expected yearly shipments of dimming ballasts to service this transformation is given on the right scale. 


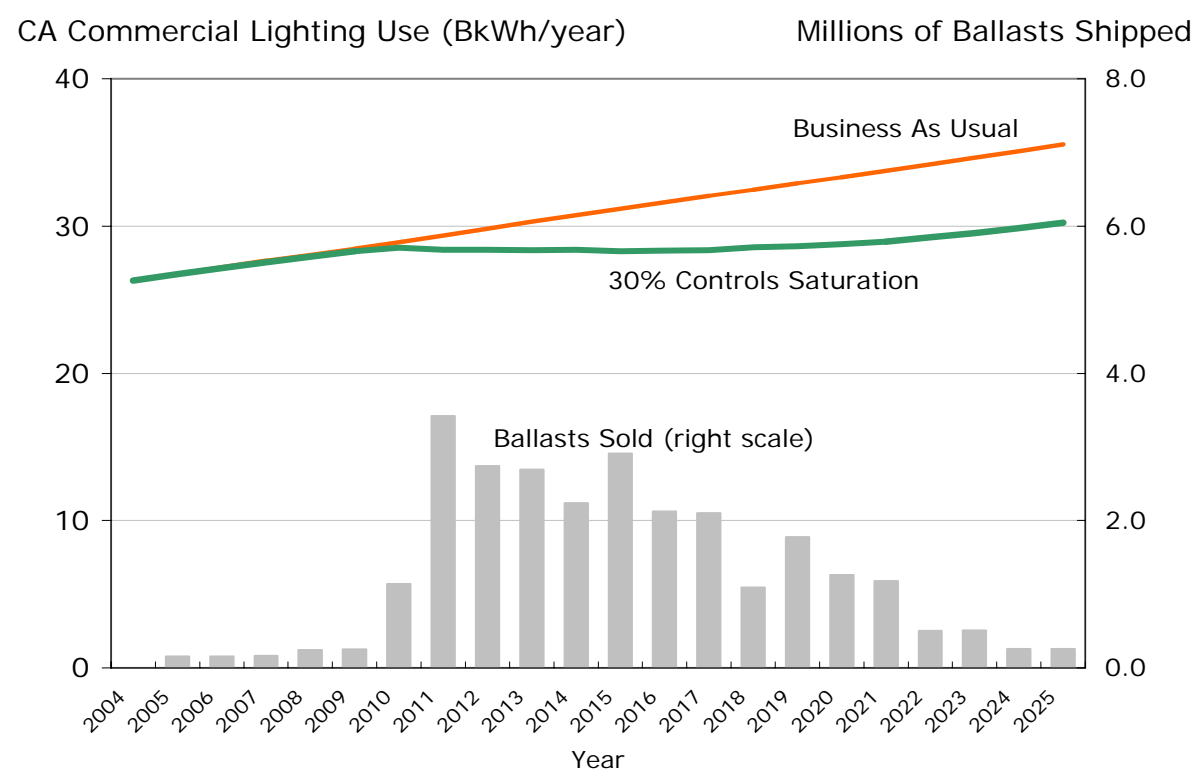

Figure 5: The estimated California energy savings (left hand scale) from deploying wireless lighting controls in $30 \%$ of CA building stock by 2025 . The expected yearly shipments of dimming ballasts to service this transformation is given on the right scale.

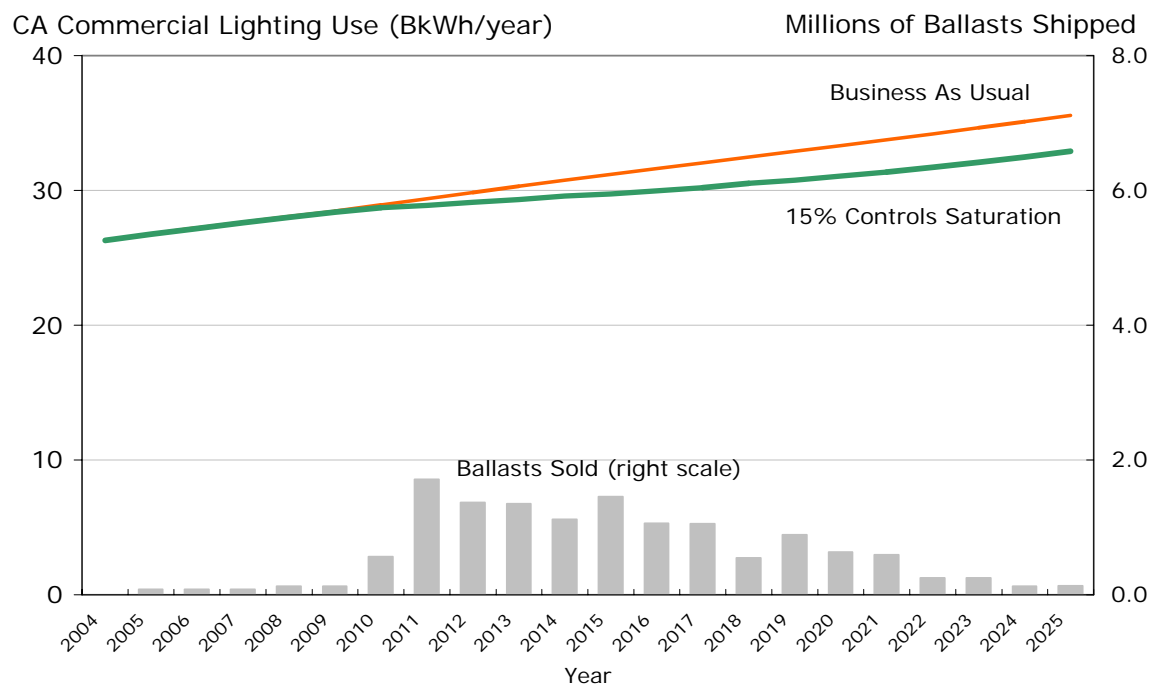

Figure 6. The estimated California energy savings (left hand scale) from deploying wireless lighting controls in $15 \%$ of CA building stock by 2025 . The expected yearly shipments of dimming ballasts to service this transformation is given on the right scale. 
Table 2 summarizes the results of this analysis for the three penetration scenarios.

\begin{tabular}{l}
$\begin{array}{l}\text { Table 2: California Energy Savings and Expected Shipments of Lighting Ballasts for Three Market } \\
\text { Penetration Scenarios }\end{array}$ \\
\hline
\end{tabular}

* $\quad \mathrm{BkWh}=$ One billion kilowatt-hours

** Value of Savings assumes $\$ 0.14 / \mathrm{kWh}$

\section{Manufacturer Benefits}

Lighting manufacturers who produce dimming ballasts and other lighting control devices will greatly expanded their market if there is a concerted effort to transform commercial building lighting to intelligent, energy-efficient lighting. In the previous section, we show that with $60 \%$ penetration of lighting controls, the lighting industry would need to produce nearly 54 million dimming ballasts by 2025 for just the State of California. During the prime years of the transformation $(2011-2022)$, the industry would sell 2 - 5 million dimming ballasts annually. This is 2 to 5 times the current one million dimming ballasts sold annually in the entire nation. Since each dimming ballast will require a wireless transceiver, this will drive up sales of wireless transceivers in California by a similar amount (an average of 3 million units between 2011 and 2022). These volumes will provide significant incentive for wireless transceiver manufacturers to lower unit prices because of greatly increased volume of sales.

Other lighting control devices, particularly sensors (light and occupancy), switches, dimmers and wireless access points could easily double the volume of wireless transceivers required (to perhaps 7 million units annually). The total value of this new market could exceed $\$ 2.6$ billion 
(approximately $\$ 1.3$ billion on wireless ballast sales and another $\$ 1.3$ billion in sales of wireless sensors, switches and access points).

\section{Summary}

This analysis indicates that a concerted national program to retrofit all buildings with wireless lighting controls over the next 20 years could exceed the California energy savings already achieved by replacing magnetic ballasts with electronic ballasts. We show that an aggressive program to achieve $60 \%$ market saturation of wireless lighting controls by 2025 would save California ratepayers $10.4 \mathrm{BkWh}$ in 2025 (a value of $\$ 1.4$ billion annually). Furthermore, the cumulative savings realized in California between 2005 and 2025 would be $100 \mathrm{BkWh}$, with an economic value of over $\$ 14$ billion. This transformation program would provide significant new business opportunities for lighting companies with cumulative expected sales of ballasts alone in the State of about $\$ 1.3$ billion.

\section{References}

[xiii] Anderson et al., Methodological Framework for Analysis of GPRA Metrics: Application to FY04 Projects in BT and WIP, April 2003, PNNL-1423

[xiv] Personal communication with Dave Anderson, Pacific Northwest National Laboratory, April 18, 2006

[xv] Bureau of the Census, Current Industrial Reports [MQ36C(97)-5; MQ335C (01)-5] 


\section{Appendix C}

Proposed Relative System Efficiency Limits for 2008 Title 24 Revision 
To: California Energy Commission Staff

October 12, 2006

From: Francis Rubinstein (Lawrence Berkeley National Laboratory)

RE:Proposed Relative System Efficiency Limits for 2008 Revision to Title 24

I propose that Staff:

- Use Relative System Efficiency (RSE) instead of Ballast Efficacy Factor (BEF) for setting voluntary efficacy limits for T-8 fluorescent lamp/ballast systems.

- Require a minimum RSE of 0.93 for dimming ballasts for T-8 fluorescent lamps.

- Consider an interim minimum RSE for 1-lamp dimming ballasts only of 0.89 .

\begin{tabular}{|llll|}
$\begin{array}{l}\text { Proposed Relative } \\
\text { System Efficiency (RSE) }\end{array}$ & $\begin{array}{l}\text { Number of T-8 Lamps } \\
\text { Per Ballast }\end{array}$ & $\begin{array}{l}\text { Equivalent Ballast } \\
\text { Factor (BEF) }\end{array}$ & Efficacy \\
\hline & 1 & 2.9 \\
0.93 & 2 & 1.45 \\
& 3 & .97 \\
0.89 & 4 & .73 \\
& 1 & 2.78 \\
\hline
\end{tabular}

The remainder of this memo provides the technical justification for above proposals (2) and (3). The justification for replacing BEF with RSE is in [Rubinstein, Technical Memorandum: Replacing Ballast Efficacy Factor (BEF) with Relative System Efficiency (RSE), November 3, 2005].

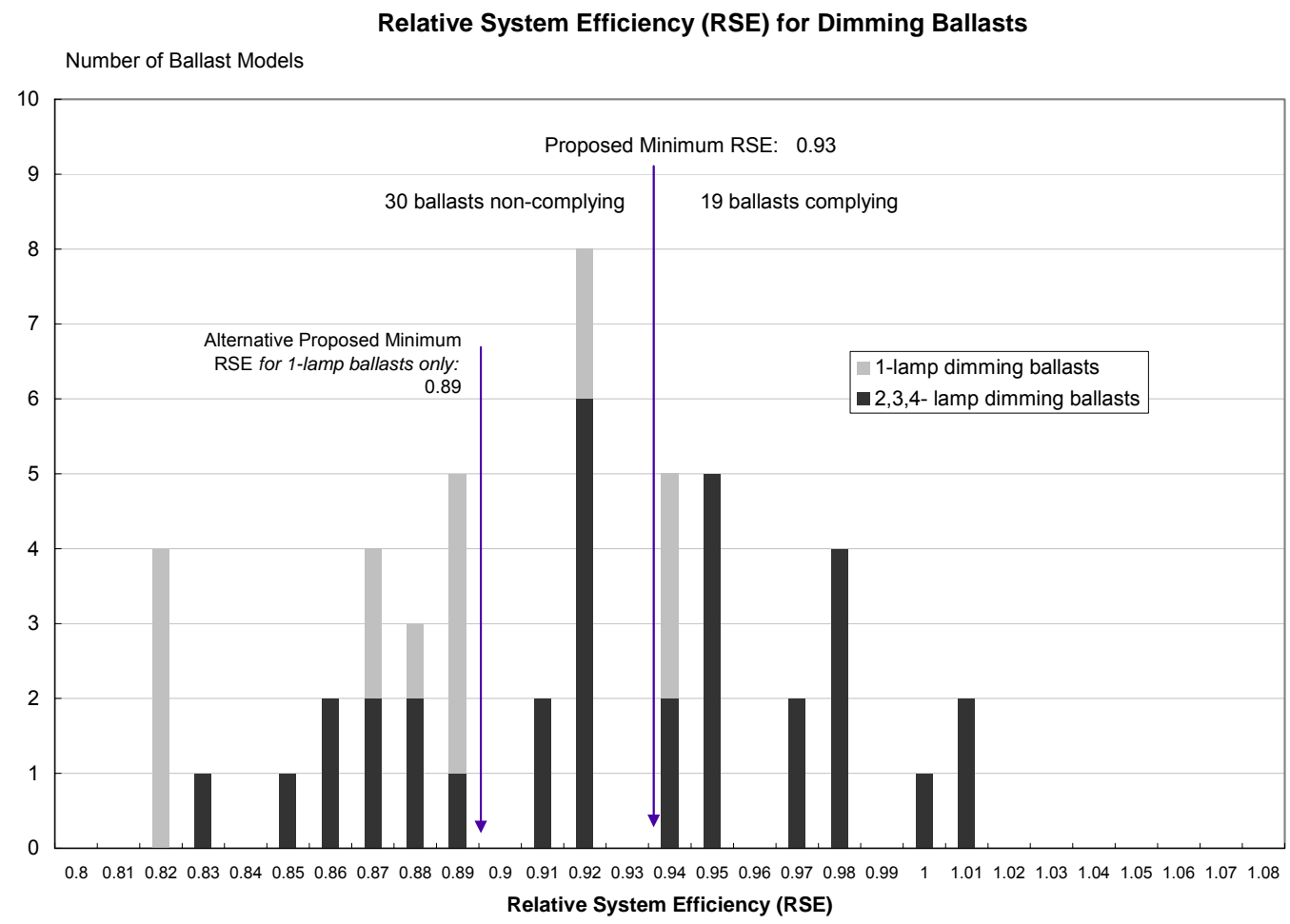

\footnotetext{
${ }^{1}$ To convert from RSE to BEF use: $\mathrm{BEF}=(\mathrm{RSE} \times 100) /(\#$ lamps $\times 32$ watts $)$
} 
The above figure shows the distribution of Relative System Efficiency (RSE) from a database of 49 dimming ballast models [(16) 1-lamp ballasts and (33) 2,3 or 4-lamp ballasts] as well as the Proposed Minimum RSE of 0.93. [Rubinstein and Kiliccote, Demand Response Lighting: A Scoping Study, Final Report to Demand Response Research Center, Draft October 1, 2006]. A minimum RSE of 0.93 would allow 19 dimming ballasts and reject 30 others.

The 1-lamp dimming ballasts have a statistically significant lower average RSE than the 2, 3, and 4-lamp dimming ballasts. (A one-tailed T-test of the difference of the means yields $t=1.68$, which rejects the hypothesis that the average RSE of the 1-lamp and 2-4 lamp ballasts are the same at higher than $98 \%$ confidence level). Because of the relatively high ballast loss for 1-lamp operation, there is some justification to set an Interim RSE of 0.89 for 1-lamp dimming ballasts only. On the other hand, there are three 1-lamp dimming ballasts in the whole sample that DO exceed the 0.93 level so manufacturers are capable of making a 1-lamp dimming ballast that complies at the higher level.

The following table provides descriptive statistics for High Performance Instant Start, Program Start and Dimming Ballasts. The table shows that the average RSE for all dimming ballasts in the sample is about $10 \%$ less than the average RSE for the new generation HP Instant Start ballasts.

\begin{tabular}{|lllllll|}
\hline & $\begin{array}{l}\text { HP Instant } \\
\text { Start } \\
\mathbf{( N = 9 5 )}\end{array}$ & $\begin{array}{l}\text { Program } \\
\text { Start } \\
\mathbf{( N = 2 1 )}\end{array}$ & $\begin{array}{l}\text { All } \\
\text { Dimming } \\
\mathbf{( N = 4 9 )}\end{array}$ & $\begin{array}{l}\text { Dimming } \\
\mathbf{2} \text { lam, } \\
\text { lamp } \\
\mathbf{( N = 3 3 )}\end{array}$ & $\begin{array}{l}\text { Dimming 1- } \\
\text { lamp } \\
\text { (N=16) }\end{array}$ \\
\hline $\begin{array}{l}\text { Number of Ballasts } \\
\text { Sample }\end{array}$ & 95 & 21 & 49 & 33 & 16 \\
$\begin{array}{l}\text { Mean RSE } \\
\text { \% Reduction in Mean RSE }\end{array}$ & $0.0 \%$ & $-5.5 \%$ & $-10.9 \%$ & $-9.5 \%$ & $-13.7 \%$ \\
$\begin{array}{l}\text { Relative to Instant Start } \\
\text { Standard Deviation }\end{array}$ & 0.02 & 0.036 & 0.049 & 0.046 & 0.044 \\
$\begin{array}{l}\text { Maximum RSE } \\
\text { Minimum RSE }\end{array}$ & 1.06 & 1.03 & 1.006 & 1.006 & 0.940 \\
\hline
\end{tabular}

By way of comparison, the proposed RSE limit of 0.93 for dimming ballasts is $9 \%$ lower than the average RSE for the HP Instant Start ballasts.

Figure 2 shows the average RSE and other descriptive statistics for 1) High Performance Instant Start, 2) Program Start and 3) Dimming Ballasts. The data for the High Performance Instant Start and Program Start Ballasts are from Consortium for Energy Efficiency [personal communication with Afroz Kahn; also see www.cee1.org]. 
Average RSEs for Dimming Ballasts Compared to High Performance Instant Start and Program Start Ballasts

Relative System Efficiency (RSE)

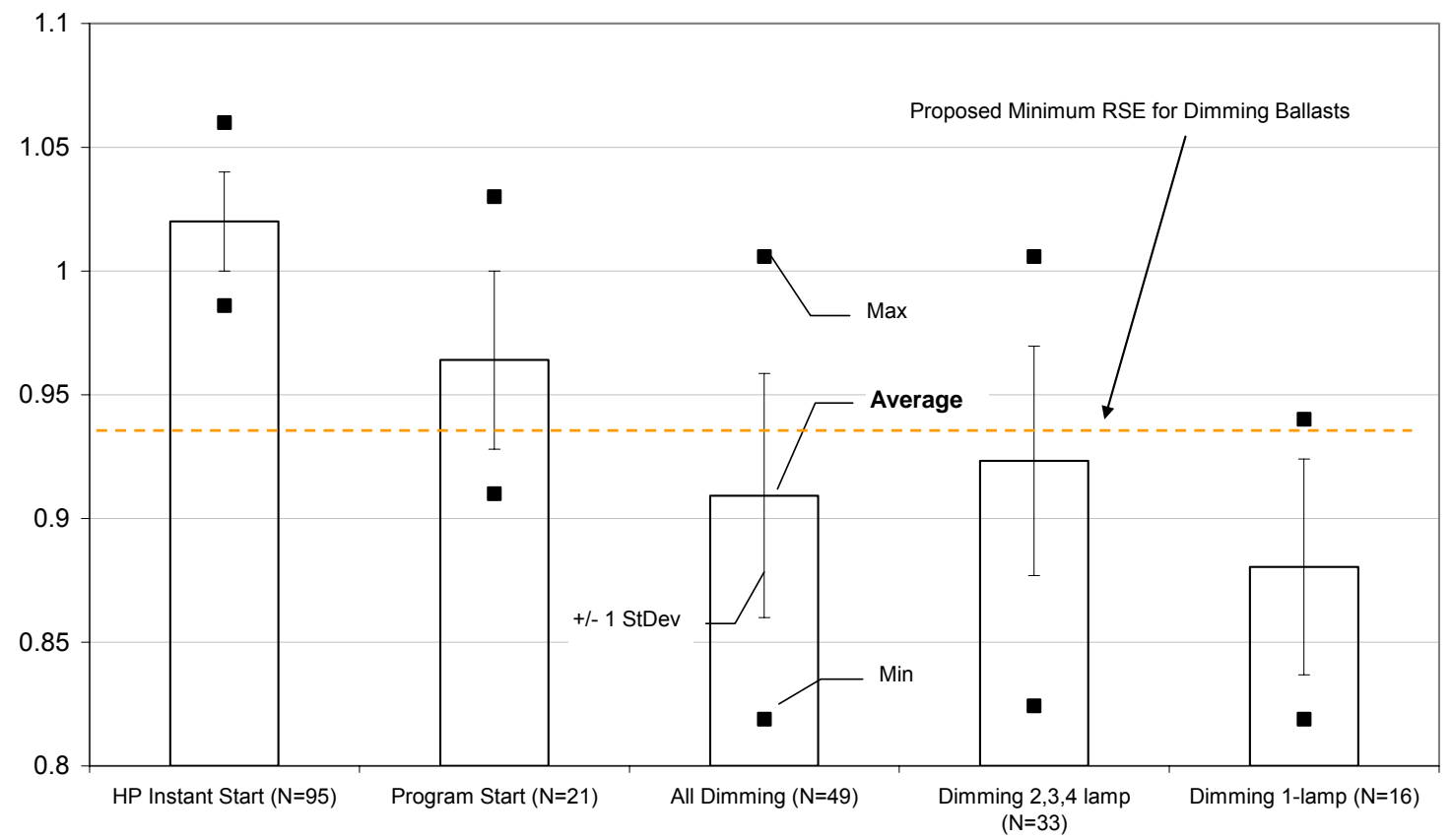

